\title{
The Genetics of Myopia
}

\author{
Milly S. Tedja, Annechien E. G. Haarman, \\ Magda A. Meester-Smoor, Virginie J. M. Verhoeven, \\ Caroline C. W. Klaver, and Stuart MacGregor
}

\section{Key Points}

- While the recent global rise of myopia prevalence is primarily attributable to environmental changes, within populations inherited factors play a large role in explaining why some individuals are affected by myopia while others are not.

- Early efforts to identify the specific genes underlying the heritability of refractive error used linkage and candidate gene designs to identify up to 50 loci and genes, although most remain unconfirmed.

M. S. Tedja · A. E. G. Haarman · M. A. Meester-Smoor

Department of Ophthalmology, Erasmus Medical Center, Rotterdam, The Netherlands

Department of Epidemiology, Erasmus Medical Center, Rotterdam, The Netherlands

V. J. M. Verhoeven

Department of Ophthalmology, Erasmus Medical Center, Rotterdam, The Netherlands

Department of Epidemiology, Erasmus Medical Center, Rotterdam, The Netherlands

Department of Clinical Genetics, Erasmus Medical Center, Rotterdam, The Netherlands

C. C. W. Klaver

Department of Ophthalmology, Erasmus Medical Center, Rotterdam, The Netherlands

Department of Epidemiology, Erasmus Medical Center, Rotterdam, The Netherlands

Department of Ophthalmology, Radboud University Medical Center,

Nijmegen, The Netherlands

S. MacGregor $(\square)$

Statistical Genetics, QIMR Berghofer Medical Research Institute,

Brisbane, QLD, Australia

e-mail: stuart.macgregor@qimrberghofer.edu.au 
- As the sample size in genome-wide association studies (GWAS) has increased, the number of implicated loci has risen steadily, with 161 variants reported in the latest meta-analysis.

- Interrogation of loci uncovered by GWAS offers insight into the molecular basis of myopia-for example, pathway analysis implicates the light induced retina-to-sclera signaling pathway in myopia development.

- Although many loci have been uncovered by GWAS, statistical modelling shows there are many more genes to find-identifying these will further illuminate the molecular pathways leading to myopia and open up new avenues for intervention.

\subsection{Introduction}

This chapter addresses the scientific exploration of the genetic architecture of myopia. Myopia is the most common eye condition worldwide and its prevalence is increasing. Changes in environmental conditions where time spent outdoors has reduced relative to previous generations are the main hypothesized culprit. Despite these environmental trends, within populations, myopia is highly heritable; genes explain up to $80 \%$ of the variance in refractive error. Initial attempts to identify myopia genes relied on family studies using linkage analysis or candidate gene approaches with limited progress. For the last decade, genome-wide association study (GWAS) approaches have predominated, ultimately resulting in the identification of hundreds of genes for refractive error and myopia, providing new insights into its molecular machinery. Thanks to these studies, it was revealed that myopia is a complex trait, with many genetic variants of small effect influencing retinal signaling, eye growth and the normal process of emmetropization. However, the genetic architecture and its molecular mechanisms are still to be clarified and while genetic risk score prediction models are improving, this knowledge must be expanded to have impact on clinical practice.

Some sections of this report follow the framework described in a recent International Myopia Institute Genetics report by Tedja et al. [1]

\subsection{Heritability}

The tendency for myopia to run in families has long been noted, suggesting genetic factors play a role in determining risk [2]. While family studies show familial aggregation, twin studies are required to reliably separate the effects of genes and familial environment [3-6]. Benchmarking of the relative contribution of genetics and environment is done by computation of heritability, the proportion of the total trait variance (here, spherical equivalent) due to additive genetic factors. Since the contributions of genes and environment can vary across human populations, heritabilities are population and 
even time specific [7, 8]. The influence of environmental variance is well illustrated in the case of the heritability study in Alaskan Eskimos, where the rapid introduction of the American school system dramatically increased the contribution of the environment. As a result heritability estimates, computed based on families where the parents are less educated relative to their offspring, were very low at this time (10\%) [7].

Across most human populations, environment is fairly constant and the estimates of spherical equivalent heritability are usually high ( 80\%) [9-11]. Although the aggregate contribution of genetic factors to variation in refractive error is high, initial studies were unable to determine the genetic architecture of myopia-that is, is myopia caused by rare mutations of large effect? Or is most variation driven by common variants, each with individually small effect on risk? With the advent of genotyping arrays, it became possible to estimate the aggregate effect of all common variants, with "array heritability" estimates of $35 \%$ from the ALSPAC study. Such estimates place a lower bound on the proportion of the heritability that is attributable to genetic variants which are common in the population. The remaining $45 \%(80 \%-35 \%)$ is likely attributable to rare genetic variants, to variants not covered by genotyping arrays or to non-additive genetic effects.

\subsection{Syndromic Myopia}

Syndromic myopia is generally monogenic and can occur within a wide spectrum of clinical presentations. This type of myopia is usually accompanied by other systemic or ocular disorders. Table 5.1 summarizes all syndromic and ocular conditions that present with myopia [12]. We are able to learn about myopia development by investigating these syndromes. For instance, several types of heritable syndromes result in extreme axial elongation, due to abnormalities in the development of connective tissue (i.e. Marfan syndrome, OMIM \#154700; Stickler syndrome, OMIM \#,108300 \#604841, \#614134, \#614284 and Ehlers-Danlos syndrome, OMIM \#225400, \#601776). Similarly, inherited retinal dystrophies lead to myopia due to defects in photoreceptors, for instance, in X-linked retinitis pigmentosa (mutations in $R P G R$-gene) and congenital stationary night blindness [13].

Interestingly, several syndromic myopia genes were found in association to other ocular traits, such as CCT (ADAMTS2, COLAA3, COL5A1, FBN1) [14], and Fuchs's dystrophy (TCF4) [15]. However, the majority of the genes causing syndromic myopia have not been linked to common forms of myopia, except for COL2A1 [16, 17] and FBN1 [18, 19]. Nevertheless, a recent study found an overrepresentation for syndromic myopia genes in GWAS studies on refractive error and myopia [20], implying their important role in myopia development.

\subsection{Linkage Studies}

Linkage studies have been successfully applied for many Mendelian disorders, although the success has been much more limited in complex traits. The linkage approach searches for cosegregation of genetic markers with the trait of interest in 
Table 5.1 Overview of syndromic forms of myopia

\begin{tabular}{|c|c|c|}
\hline Syndrome & $\begin{array}{l}\text { Gene and inheritance } \\
\text { pattern }\end{array}$ & Ocular phenotype other than myopia \\
\hline \multicolumn{3}{|c|}{ (A) Syndromes associated with myopia and associated ocular phenotype } \\
\hline $\begin{array}{l}\text { Acromelic frontonasal } \\
\text { dysostosis }\end{array}$ & ZSWIM6 (AD) & $\begin{array}{l}\text { Telecanthus, ptosis (some patients), corneal } \\
\text { dermoid cyst (rare), glaucoma (rare), } \\
\text { segmental optic nerve hypoplasia (rare), } \\
\text { persistent primary vitreous (rare) }\end{array}$ \\
\hline Alagille syndrome & $J A G 1(A D)$ & $\begin{array}{l}\text { Deep-set eyes, hypertelorism, upslanting } \\
\text { palpebral fissures, posterior embryotoxon, } \\
\text { anterior chamber anomalies, eccentric or } \\
\text { ectopic pupils, chorioretinal atrophy, band } \\
\text { keratopathy, cataracts, retinal pigment } \\
\text { clumping, Axenfeld anomaly, microcornea, } \\
\text { choroidal folds, strabismus, anomalous } \\
\text { optic disc }\end{array}$ \\
\hline Alport syndrome & $\begin{array}{l}\text { COLAA5 }(X L D) \\
\text { COLAA3 }(A R / A D)\end{array}$ & $\begin{array}{l}\text { Anterior lenticonus, lens opacities, } \\
\text { cataracts, pigmentary changes ("flecks") in } \\
\text { the perimacular region, corneal endothelial } \\
\text { vesicles, corneal erosions }\end{array}$ \\
\hline Angelman syndrome & UBE3A (IP); CH & $\begin{array}{l}\text { Strabismus (most frequently exotropia), } \\
\text { ocular hypopigmentation, refractive errors } \\
\text { (astigmatism, hyperopia, myopia) }\end{array}$ \\
\hline Bardet-Biedl syndrome & $\begin{array}{l}\text { ARL6; BBS1; BBS2; } \\
\text { BBS4; BBS5;BBS7; } \\
\text { BBS9;BBS10; } \\
\text { BBS12; CEP290; } \\
\text { LZTFL1; MKKS; } \\
\text { MKS1; SDCCAG8; } \\
\text { TMEM67; TRIM32; } \\
\text { TTC8; WDPCP }(A R)\end{array}$ & $\begin{array}{l}\text { Rod-cone dystrophy onset by end of } 2 \mathrm{nd} \\
\text { decade, retinitis pigmentosa, retinal } \\
\text { degeneration, strabismus, cataracts }\end{array}$ \\
\hline Beals syndrome & $F B N 2(A D)$ & Ectopia lentis \\
\hline $\begin{array}{l}\text { Beaulieu-Boycott-Innes } \\
\text { syndrome }\end{array}$ & THOC6 $(A R)$ & $\begin{array}{l}\text { Deep-set eyes, short palpebral fissures, } \\
\text { upslanting palpebral fissures }\end{array}$ \\
\hline Bohring-Opitz syndrome & $A S X L 1(A D)$ & $\begin{array}{l}\text { Prominent eyes, hypoplastic orbital ridges, } \\
\text { hypertelorism, upslanting palpebral } \\
\text { fissures, strabismus, retinal abnormalities, } \\
\text { optic nerve abnormalities }\end{array}$ \\
\hline $\begin{array}{l}\text { Bone fragility and } \\
\text { contractures; arterial } \\
\text { rupture and deafness }\end{array}$ & PLOD3 $(A R)$ & Shallow orbits, cataracts \\
\hline $\begin{array}{l}\text { Branchiooculofacial } \\
\text { syndrome }\end{array}$ & $T F A P 2 A(A D)$ & $\begin{array}{l}\text { Lacrimal sac fistula, orbital dermoid cyst, } \\
\text { iris pigment epithelial cyst, combined } \\
\text { hamartoma of the retina and retinal } \\
\text { pigment epithelium, upslanting palpebral } \\
\text { fissures, telecanthus, hypertelorism, ptosis, } \\
\text { lacrimal duct obstruction, iris coloboma, } \\
\text { retinal coloboma, microphthalmia, } \\
\text { anophthalmia, cataract, strabismus }\end{array}$ \\
\hline
\end{tabular}


Table 5.1 (continued)

\begin{tabular}{|c|c|c|}
\hline Syndrome & $\begin{array}{l}\text { Gene and inheritance } \\
\text { pattern }\end{array}$ & Ocular phenotype other than myopia \\
\hline $\begin{array}{l}\text { Cardiofaciocutaneous } \\
\text { syndrome }\end{array}$ & $M A P 2 K 2(A D)$ & $\begin{array}{l}\text { Ptosis, nystagmus, strabismus, } \\
\text { downslanting palpebral fissures, } \\
\text { hypertelorism, exophthalmos, epicanthal } \\
\text { folds, optic nerve dysplasia, oculomotor } \\
\text { apraxia, loss of visual acuity, absence of } \\
\text { eyebrows, absence of eyelashes }\end{array}$ \\
\hline Cohen syndrome & $\operatorname{VPS} 13 B(A R)$ & $\begin{array}{l}\text { Downslanting palpebral fissures, almond- } \\
\text { shaped eyes, chorioretinal dystrophy, } \\
\text { decreased visual acuity, optic atrophy }\end{array}$ \\
\hline $\begin{array}{l}\text { Cornelia de Lange } \\
\text { syndrome }\end{array}$ & $\begin{array}{l}\text { NIPBL }(A D) \\
H D A C 8(X L D)\end{array}$ & Synophrys, long curly eyelashes, ptosis \\
\hline Cowden syndrome & PTEN (AD) & Cataract, angioid streaks \\
\hline $\begin{array}{l}\text { Cranioectodermal } \\
\text { dysplasia }\end{array}$ & IFT122(AR) & $\begin{array}{l}\text { Telecanthus, hypotelorism, epicanthal } \\
\text { folds, myopia ( } 1 \text { patient), nystagmus ( } 1 \\
\text { patient), retinal dystrophy ( } 1 \text { patient) }\end{array}$ \\
\hline Cutis laxa & $\begin{array}{l}\text { ATP6VOA2; } \\
\text { ALDH18A1 }(A R)\end{array}$ & $\begin{array}{l}\text { Downslanting palpebral fissures, } \\
\text { strabismus }\end{array}$ \\
\hline Danon disease & $L A M P 2(X L D)$ & $\begin{array}{l}\text { Moderate central loss of visual acuity in } \\
\text { males, normal to near-normal visual acuity } \\
\text { in carrier females, fine lamellar white } \\
\text { opacities on slit lamp exam in carrier } \\
\text { females, near complete loss of peripheral } \\
\text { retinal pigment in males, peppered } \\
\text { pigmentary mottling of peripheral retinal } \\
\text { pigment in carrier females, nonspecific } \\
\text { changes on electroretinogram in carrier } \\
\text { females }\end{array}$ \\
\hline Deafness and myopia & SLITRK6 $(A R)$ & High myopia \\
\hline $\begin{array}{l}\text { Desanto-Shinawi } \\
\text { syndrome }\end{array}$ & $W A C(A D)$ & $\begin{array}{l}\text { Hypertelorism, downslanting palpebral } \\
\text { issues, synophrys, deep-set eyes, } \\
\text { astigmatism, strabismus }\end{array}$ \\
\hline Desbuquois dysplasia & CANT1 $(A R)$ & $\begin{array}{l}\text { Prominent eyes, bulging eyes, congenital } \\
\text { glaucoma }\end{array}$ \\
\hline $\begin{array}{l}\text { Donnai-Barrow } \\
\text { syndrome }\end{array}$ & $L R P 2(A R)$ & $\begin{array}{l}\text { Hypertelorism, high myopia, loss of vision, } \\
\text { iris coloboma, iris hypoplasia, cataract, } \\
\text { enlarged globes, downslanting palpebral } \\
\text { fissures, underorbital skin creases, retinal } \\
\text { detachment, retinal dystrophy, prominent } \\
\text { eyes }\end{array}$ \\
\hline DOORS & $T B C 1 D 24(A R)$ & $\begin{array}{l}\text { Optic atrophy, blindness, high myopia, } \\
\text { cataracts }\end{array}$ \\
\hline Ehlers-Danlos syndrome & $\begin{array}{l}\text { COL5A1 (AD); } \\
\text { PLOD1 (AR); } \\
\text { CHST14 (AR); } \\
\text { ADAMTS2 }(A R) \\
\text { B3GALT6 }(A R) ; \\
\text { FKBP14 }(A R)\end{array}$ & $\begin{array}{l}\text { Blue sclerae, ectopia lentis, epicanthal } \\
\text { folds }\end{array}$ \\
\hline
\end{tabular}


Table 5.1 (continued)

\begin{tabular}{|c|c|c|}
\hline Syndrome & $\begin{array}{l}\text { Gene and inheritance } \\
\text { pattern }\end{array}$ & Ocular phenotype other than myopia \\
\hline Emanuel syndrome & $\mathrm{CH}$ & $\begin{array}{l}\text { Hooded eyelids, deep-set eyes, upslanting } \\
\text { palpebral fissures, strabismus }\end{array}$ \\
\hline Fibrochondrogenesis & COL11A1 (AR) & - \\
\hline $\begin{array}{l}\text { Gyrate atrophy of } \\
\text { choroid and retina with/ } \\
\text { without ornithinemia }\end{array}$ & $O A T(A R)$ & $\begin{array}{l}\text { Progressive chorioretinal degeneration, } \\
\text { night blindness (onset in first decade, } \\
\text { progressive loss of peripheral vision, } \\
\text { blindness (onset in fourth or fifth decade), } \\
\text { posterior subcapsular cataracts (onset in } \\
\text { second or third decade) }\end{array}$ \\
\hline Hamamy syndrome & $\operatorname{IRX} 5(A R)$ & $\begin{array}{l}\text { Severe hypertelorism, laterally sparse } \\
\text { eyebrows, myopia (progressive severe) }\end{array}$ \\
\hline Homocystinuria & $C B S(A R)$ & Ectopia lentis, glaucoma \\
\hline $\begin{array}{l}\text { Joint laxity; short stature; } \\
\text { myopia }\end{array}$ & GZF1 (AR) & $\begin{array}{l}\text { Exophthalmos, severe myopia, retinal } \\
\text { detachment (some patients), iris coloboma } \\
\text { (some patients), chorioretinal coloboma } \\
\text { (some patients), glaucoma (1 patient) }\end{array}$ \\
\hline $\begin{array}{l}\text { Kaufman } \\
\text { oculocerebrofacial } \\
\text { syndrome }\end{array}$ & $U B E 3 B(A R)$ & $\begin{array}{l}\text { Blepharophimosis, ptosis, upward-slanting } \\
\text { palpebral fissures, telecanthus, } \\
\text { hypertelorism, astigmatism, strabismus, } \\
\text { mild }\end{array}$ \\
\hline Kenny-Caffey syndrome & FAM111A $(A D)$ & $\begin{array}{l}\text { Hyperopia (not myopia), microphthalmia, } \\
\text { papilledema, corneal and retinal } \\
\text { calcification, congenital cataracts (rare) }\end{array}$ \\
\hline Kniest dysplasia & COL2A1 $(A D)$ & $\begin{array}{l}\text { Retinal detachment, cataracts, prominent } \\
\text { eyes }\end{array}$ \\
\hline Knobloch syndrome & COL18A1 (AR) & $\begin{array}{l}\text { High myopia, vitreoretinal degeneration, } \\
\text { retinal detachment (childhood), congenital } \\
\text { cataract, syneresis, vitreous attachment at } \\
\text { the disc, persistent foetal hyaloid } \\
\text { vasculature, peripapillary atrophy, phthisis } \\
\text { bulbi, band keratopathy, macular } \\
\text { hypoplasia, irregular white dots at the } \\
\text { vitreoretinal interface, visual loss, } \\
\text { nystagmus }\end{array}$ \\
\hline Lamb-Shaffer syndrome & SOX5 $(A D)$ & $\begin{array}{l}\text { Downslanting palpebral fissures, epicanthal } \\
\text { folds, strabismus }\end{array}$ \\
\hline $\begin{array}{l}\text { Lethal congenital } \\
\text { contracture syndrome }\end{array}$ & $E R B B 3(A R)$ & $\begin{array}{l}\text { High myopia, degenerative } \\
\text { vitreoretinopathy }\end{array}$ \\
\hline Leukodystrophy & $\begin{array}{l}\text { POLRIC; POLR } 3 A ; \\
\text { POLR } 3 B ; \text { GJC2 } \\
(A R)\end{array}$ & - \\
\hline $\begin{array}{l}\text { Linear skin defects with } \\
\text { multiple congenital } \\
\text { anomalies }\end{array}$ & $\begin{array}{l}\text { NDUFB11;COX7B } \\
(X L D)\end{array}$ & $\begin{array}{l}\text { Lacrimal duct atresia, nystagmus, } \\
\text { strabismus }\end{array}$ \\
\hline Loeys-Dietz syndrome & $\begin{array}{l}\text { TGFBR1; TGFBR2 } \\
(A D)\end{array}$ & $\begin{array}{l}\text { Hypertelorism, exotropia, blue sclerae, } \\
\text { proptosis }\end{array}$ \\
\hline
\end{tabular}


Table 5.1 (continued)

\begin{tabular}{|c|c|c|}
\hline Syndrome & $\begin{array}{l}\text { Gene and inheritance } \\
\text { pattern }\end{array}$ & Ocular phenotype other than myopia \\
\hline $\begin{array}{l}\text { Macrocephaly/ } \\
\text { megalencephaly } \\
\text { syndrome }\end{array}$ & TBC1D7 (AR) & Astigmatism \\
\hline Marfan syndrome & FBN1 $(A D)$ & $\begin{array}{l}\text { Enophthalmos, ectopia lentis increased } \\
\text { axial globe length, corneal flatness, retinal } \\
\text { detachment, iris hypoplasia, early } \\
\text { glaucoma, early cataracts, downslanting } \\
\text { palpebral fissures, trabeculodysgenesis, } \\
\text { primary (some patients), strabismus (some } \\
\text { patients), exotropia (some patients), } \\
\text { esotropia (rare), hypertropia (rare) }\end{array}$ \\
\hline Marshall syndrome & COL11A1 (AD) & $\begin{array}{l}\text { congenital cataracts, esotropia, retinal } \\
\text { detachment, glaucoma, lens dislocation, } \\
\text { vitreoretinal degeneration, hypertelorism, } \\
\text { epicanthal folds }\end{array}$ \\
\hline $\begin{array}{l}\text { Microcephaly with/ } \\
\text { without } \\
\text { chorioretinopathy; } \\
\text { lymphedema; and/or } \\
\text { mental retardation }\end{array}$ & KIF11 (AD) & $\begin{array}{l}\text { Upslanting palpebral fissures, downslanting } \\
\text { palpebral fissures (some patients), } \\
\text { epicanthal folds (some patients), } \\
\text { nystagmus, reduced visual acuity, } \\
\text { hypermetropia, myopic astigmatism, } \\
\text { hypermetropic astigmatism, corneal } \\
\text { opacity, microcornea, microphthalmia, } \\
\text { cataract, retrolenticular fibrotic mass, } \\
\text { chorioretinopathy, retinal folds, falciform } \\
\text { retinal folds, retinal detachment, temporal } \\
\text { dragging of optic disc, retinal pigment } \\
\text { changes (some patients), optic atrophy } \\
\text { (uncommon) }\end{array}$ \\
\hline $\begin{array}{l}\text { Mohr-Tranebjaerg } \\
\text { syndrome }\end{array}$ & TIMM $8 A(X L R)$ & $\begin{array}{l}\text { Photophobia, cortical blindness, decreased } \\
\text { visual acuity, constricted visual fields, } \\
\text { abnormal electroretinogram }\end{array}$ \\
\hline Mucolipidosis & GNPTAG $(A R)$ & Fine corneal opacities \\
\hline Muscular dystrophy & $\begin{array}{l}\text { TRAPPC11; POMT; } \\
\text { POMT1; POMT2; } \\
\text { POMGNT1; } \\
\text { B3GALNT2; FKRP; } \\
\text { DAG1; FKTN }(A R)\end{array}$ & $\begin{array}{l}\text { Cataracts, strabismus, alacrima (some } \\
\text { patients) }\end{array}$ \\
\hline Nephrotic syndrome & $L A M B 2(A R)$ & $\begin{array}{l}\text { Nystagmus, strabismus, microcoria, } \\
\text { aplasia/atrophy of the dilatator pupillae } \\
\text { muscle, hypoplasia of the iris and ciliary } \\
\text { body, lenticonus posterior, blindness, } \\
\text { decreased or absent laminin beta-2 } \\
\text { immunoreactivity in tissues of the anterior } \\
\text { eye }\end{array}$ \\
\hline
\end{tabular}


Table 5.1 (continued)

\begin{tabular}{|c|c|c|}
\hline Syndrome & $\begin{array}{l}\text { Gene and inheritance } \\
\text { pattern }\end{array}$ & Ocular phenotype other than myopia \\
\hline Noonan syndrome & $\begin{array}{l}A 2 M L 1 ; B R A F ; \\
C B L ; H R A S ; \text { KRAS; } \\
\text { MAP } 2 K 1 ; M A P 2 K 2 ; \\
\text { NRAS; PTPN11; } \\
\text { RAF1; RIT1; SOS1; } \\
\text { SHOC2; SPRED1 } \\
(A D)\end{array}$ & $\begin{array}{l}\text { Ptosis, hypertelorism, downslanting } \\
\text { palpebral fissures, epicanthal folds, } \\
\text { blue-green irides }\end{array}$ \\
\hline Oculocutaneous albinism & $T Y R(A R)$ & $\begin{array}{l}\text { Absent pigment in iris and retina, } \\
\text { translucent iris, pink irides (childhood), } \\
\text { blue-gray irides (adult), choroidal vessels } \\
\text { visible, foveal hypoplasia, decreased visual } \\
\text { acuity, strabismus, nystagmus, } \\
\text { photophobia, high refractive errors } \\
\text { (hyperopia, myopia, with-the-rule } \\
\text { astigmatism), albinotic optic disc, } \\
\text { misrouting of the optic nerves at the } \\
\text { chiasm, absent stereopsis due to anomalous } \\
\text { decussation at the optic chiasm, positive } \\
\text { angle kappa (appearance of exotropia but } \\
\text { no shift on cover test), asymmetric visual } \\
\text { evoked potentials }\end{array}$ \\
\hline $\begin{array}{l}\text { Oculodentodigital } \\
\text { dysplasia }\end{array}$ & GJA1 (AR) & $\begin{array}{l}\text { Hypoplastic eyebrows, sparse eyelashes, } \\
\text { telecanthus, short palpebral fissures, } \\
\text { downslanting palpebral fissures, } \\
\text { microphthalmia, microcornea, cataract, } \\
\text { persistent pupillary membrane }\end{array}$ \\
\hline $\begin{array}{l}\text { Pallister-Killian } \\
\text { syndrome }\end{array}$ & $\mathrm{CH}$ & $\begin{array}{l}\text { Sparse eyebrows, sparse eyelashes, } \\
\text { upslanting palpebral fissures, } \\
\text { hypertelorism, ptosis, strabismus, } \\
\text { epicanthal folds, cataracts, exophthalmos }\end{array}$ \\
\hline Papillorenal syndrome & $P A X 2(A D)$ & $\begin{array}{l}\text { Retinal coloboma, optic nerve anomalies } \\
\text { (coloboma, gliosis, absent optic nerve } \\
\text { head), optic disc anomalies (dysplasia, } \\
\text { excavation, hyperplasia, morning glory } \\
\text { optic disc, hypoplasia), orbital cysts, } \\
\text { microphthalmia, abnormal retinal pigment } \\
\text { epithelium, abnormal retinal vessels, } \\
\text { chorioretinal degeneration, retinal } \\
\text { detachment (rare). retinal staphyloma } \\
\text { (rare), retinal edema (rare), macular } \\
\text { degeneration (rare), papillomacular } \\
\text { detachment (rare), hyperpigmentation of } \\
\text { the macula (rare), cystic degeneration of } \\
\text { the macula (rare), posterior lens luxation } \\
\text { (rare), lens opacity (rare) }\end{array}$ \\
\hline
\end{tabular}


Table 5.1 (continued)

\begin{tabular}{|c|c|c|}
\hline Syndrome & $\begin{array}{l}\text { Gene and inheritance } \\
\text { pattern }\end{array}$ & Ocular phenotype other than myopia \\
\hline Peters-plus syndrome & $B 3 G L C T(A R)$ & $\begin{array}{l}\text { Hypertelorism, Peter's anomaly, anterior } \\
\text { chamber cleavage disorder, nystagmus, } \\
\text { ptosis, glaucoma, upslanting palpebral } \\
\text { fissures, cataract, iris coloboma, retinal } \\
\text { coloboma }\end{array}$ \\
\hline Pitt-Hopkins syndrome & TCF4 (AD) & $\begin{array}{l}\text { Deep-set eyes, strabismus, astigmatism, } \\
\text { upslanting palpebral fissures }\end{array}$ \\
\hline $\begin{array}{l}\text { Pontocerebellar } \\
\text { hypoplasia }\end{array}$ & CHMP1A $(A R)$ & $\begin{array}{l}\text { Astigmatism, esotropia, strabismus, } \\
\text { hyperopia, nystagmus (some patients), } \\
\text { cortical visual impairment (some patients), } \\
\text { poor visual tracking (some patients) }\end{array}$ \\
\hline $\begin{array}{l}\text { Poretti-Boltshauser } \\
\text { syndrome }\end{array}$ & LAMA1 (AR) & $\begin{array}{l}\text { Strabismus, amblyopia, oculomotor } \\
\text { apraxia, nystagmus, retinal atrophy, retinal } \\
\text { dystrophy, retinal dysfunction, macular } \\
\text { heterotopia }\end{array}$ \\
\hline Prader-Willi syndrome & $\begin{array}{l}N D N(\mathrm{PC}) ; S N R P N \\
\text { (IP); CH }\end{array}$ & $\begin{array}{l}\text { Almond-shaped eyes, strabismus, } \\
\text { upslanting palpebral fissures, hyperopia }\end{array}$ \\
\hline $\begin{array}{l}\text { Pseudoxanthoma } \\
\text { elasticum }\end{array}$ & $A B C C 6(A R)$ & $\begin{array}{l}\text { Peau d'orange retinal changes (yellow- } \\
\text { mottled retinal hyperpigmentation), angioid } \\
\text { streaks of the retina ( } 85 \% \text { of patients), } \\
\text { macular degeneration, visual impairment } \\
\text { ( } 50-70 \% \text { of patients), central vision loss, } \\
\text { colloid bodies, retinal haemorrhage, } \\
\text { choroidal neovascularization, optic head } \\
\text { drusen (yellowish-white irregularities of } \\
\text { optic disc), owl's eyes (paired } \\
\text { hyperpigmented spots) }\end{array}$ \\
\hline Renal hypomagnesemia & $\begin{array}{l}\text { CLDN16; CLDN19 } \\
(A R)\end{array}$ & $\begin{array}{l}\text { Strabismus, nystagmus, hyperopia, } \\
\text { astigmatism }\end{array}$ \\
\hline SADDAN & FGFR3 (AD) & High myopia, exotropia \\
\hline Schaaf-Yang syndrome & $M A G E L 2(A D)$ & $\begin{array}{l}\text { Esotropia, strabismus, almond-shaped eyes, } \\
\text { short palpebral fissures, bushy eyebrows }\end{array}$ \\
\hline $\begin{array}{l}\text { Schimke immunoosseous } \\
\text { dysplasia }\end{array}$ & SMARCAL1 (AR) & Corneal opacities, astigmatism \\
\hline $\begin{array}{l}\text { Schuurs-Hoeijmakers } \\
\text { syndrome }\end{array}$ & PACS1 (AD) & $\begin{array}{l}\text { Full, arched eyebrows, long eyelashes, } \\
\text { hypertelorism, downslanting palpebral } \\
\text { fissures, ptosis, nystagmus, strabismus }\end{array}$ \\
\hline $\begin{array}{l}\text { Schwartz-Jampel } \\
\text { syndrome }\end{array}$ & $H S P G 2(A R)$ & $\begin{array}{l}\text { Narrow palpebral fissures, } \\
\text { blepharophimosis, cataract, microcornea, } \\
\text { long eyelashes in irregular rows, ptosis }\end{array}$ \\
\hline Sengers syndrome & $A G K(A R)$ & Cataracts (infantile), strabismus, glaucoma \\
\hline $\begin{array}{l}\text { Short stature; hearing } \\
\text { loss; retinitis pigmentosa } \\
\text { and distinctive facies }\end{array}$ & EXOSC2 (AR) & $\begin{array}{l}\text { Deep-set eyes, short palpebral fissures, } \\
\text { upslanting palpebral fissures, retinitis } \\
\text { pigmentosa ( } 2 \text { patients), corneal dystrophy } \\
\text { (2 patients, young-adult onset), glaucoma } \\
\text { (1 patient), nystagmus (1 patient), } \\
\text { strabismus (1 patient) }\end{array}$ \\
\hline
\end{tabular}


Table 5.1 (continued)

\begin{tabular}{|c|c|c|}
\hline Syndrome & $\begin{array}{l}\text { Gene and inheritance } \\
\text { pattern }\end{array}$ & Ocular phenotype other than myopia \\
\hline $\begin{array}{l}\text { Short stature; optic nerve } \\
\text { atrophy; and Pelger-Huet } \\
\text { anomaly }\end{array}$ & $N B A S(A R)$ & $\begin{array}{l}\text { Thick and bush eyebrows, small orbits, } \\
\text { bilateral exophthalmos, epicanthus, } \\
\text { bilateral optic nerve atrophy, non- } \\
\text { progressive decreased visual acuity, (in) } \\
\text { complete achromatopsia, strabismus (some } \\
\text { patients), hypertelorism (some patients), } \\
\text { hypermetropia (rare), pigmented nevus } \\
\text { (rare) }\end{array}$ \\
\hline SHORT syndrome & PIK3RI (AD) & $\begin{array}{l}\text { Deep-set eyes, Rieger anomaly, } \\
\text { telecanthus, glaucoma, megalocornea, } \\
\text { cataracts }\end{array}$ \\
\hline $\begin{array}{l}\text { Short-rib thoracic } \\
\text { dysplasia with/without } \\
\text { polydactyly }\end{array}$ & WDR19 (AR) & $\begin{array}{l}\text { Cataracts, attenuated arteries, macular } \\
\text { anomalies }\end{array}$ \\
\hline $\begin{array}{l}\text { Shprintzen-Goldberg } \\
\text { syndrome }\end{array}$ & $S K I(A D)$ & $\begin{array}{l}\text { Telecanthus, hypertelorism, proptosis, } \\
\text { strabismus, downslanting palpebral } \\
\text { fissures, ptosis, shallow orbits }\end{array}$ \\
\hline $\begin{array}{l}\text { Singleton-Merten } \\
\text { syndrome }\end{array}$ & IFIHI $(A D)$ & Glaucoma \\
\hline $\begin{array}{l}\text { Small vessel brain } \\
\text { disease with/without } \\
\text { ocular anomalies }\end{array}$ & COL4A1 (AD) & $\begin{array}{l}\text { Retinal arteriolar tortuosity, } \\
\text { hypopigmentation of the fundus, episodic } \\
\text { scotomas, episodic blurred vision, } \\
\text { amblyopia ( } 1 \text { family), strabismus ( } 1 \\
\text { family), high intraocular pressure ( } 1 \\
\text { family). Reported in some patients: } \\
\text { astigmatism, hyperopia, congenital } \\
\text { cataracts, prominent or irregular Schwalbe } \\
\text { line, iridocorneal synechiae, Axenfeld- } \\
\text { Rieger anomalies, corneal opacities, } \\
\text { microphthalmia, microcornea, iris } \\
\text { hypoplasia, corectopia. Rare: decreased } \\
\text { visual acuity, glaucoma, corneal } \\
\text { neovascularization, polycoria, } \\
\text { iridogoniodysgenesis, macular } \\
\text { haemorrhage and Fuchs spots, peripapillary } \\
\text { atrophy, choroidal atrophy }\end{array}$ \\
\hline $\begin{array}{l}\text { Smith-Magenis } \\
\text { syndrome }\end{array}$ & $R A I 1(A D)$ & - \\
\hline $\begin{array}{l}\text { Spastic paraplegia and } \\
\text { psychomotor retardation } \\
\text { with or without seizures }\end{array}$ & HACE1 $(A R)$ & $\begin{array}{l}\text { Strabismus, retinal dystrophy (some } \\
\text { patients) }\end{array}$ \\
\hline $\begin{array}{l}\text { Split hand/foot } \\
\text { malformation }\end{array}$ & $\mathrm{CH}$ & - \\
\hline Stickler syndrome & $\begin{array}{l}\text { COL2A1 }(A D) \\
\text { COL11A1 } \\
\text { COL9A } 1 \text { (AR); } \\
\text { COL9A2 }(A R)\end{array}$ & $\begin{array}{l}\text { Retinal detachment, blindness, occasional } \\
\text { cataracts, glaucoma, membranous (type I) } \\
\text { vitreous phenotype }\end{array}$ \\
\hline
\end{tabular}


Table 5.1 (continued)

\begin{tabular}{|c|c|c|}
\hline Syndrome & $\begin{array}{l}\text { Gene and inheritance } \\
\text { pattern }\end{array}$ & Ocular phenotype other than myopia \\
\hline $\begin{array}{l}\text { Syndromic mental } \\
\text { retardation }\end{array}$ & $\begin{array}{l}\text { SETD5 (AD); MBD5 } \\
\text { (AD); USP9X } \\
\text { (XLD); NONO } \\
\text { (XLR); RPL10 } \\
\text { (XLR); SMS (XLR); } \\
\text { ELOVL4 (AR); } \\
\text { KDM5C (XLR) }\end{array}$ & $\begin{array}{l}\text { Synophrys, eyebrow abnormalities, } \\
\text { upslanting and short palpebral fissures, } \\
\text { epicanthal folds, mild hypertelorism, } \\
\text { strabismus, cataracts, hypermetropia, } \\
\text { astigmatism, poor vision }\end{array}$ \\
\hline $\begin{array}{l}\text { Syndromic } \\
\text { microphthalmia }\end{array}$ & $O T X 2 ; B M P 4(A D)$ & $\begin{array}{l}\text { Uni- or bilateral microphthalmia, uni- or } \\
\text { bilateral anophthalmia, coloboma, } \\
\text { microcornea, cataract, retinal dystrophy, } \\
\text { optic nerve hypoplasia or agenesis }\end{array}$ \\
\hline Temtamy syndrome & C12orf57 (AR) & $\begin{array}{l}\text { Hypertelorism. "key-hole" iris, retina and } \\
\text { choroid coloboma, dislocated lens } \\
\text { (upward), downslanting palpebral fissures, } \\
\text { arched eyebrows }\end{array}$ \\
\hline White-Sutton syndrome & POGZ $(A D)$ & $\begin{array}{l}\text { Visual abnormalities, strabismus, } \\
\text { astigmatism, hyperopia, optic atrophy, } \\
\text { rod-cone dystrophy, cortical blindness }\end{array}$ \\
\hline $\begin{array}{l}\text { Zimmermann-Laband } \\
\text { syndrome }\end{array}$ & KCNH1 $(A D)$ & Thick eyebrows, synophrys, cataracts \\
\hline
\end{tabular}

$A D$ autosomal dominant, $A R$ autosomal recessive, $X L R \times$ linked recessive, $X L D \mathrm{X}$ linked dominant, $\mathrm{CH}$ chromosomal, IP imprinting defect

Table 5.1A Ocular conditions associated with myopia

\begin{tabular}{|c|c|}
\hline Ocular condition & Gene and inheritance pattern \\
\hline Achromatopsia & $C N G B 3(A R)$ \\
\hline Aland Island eye disease & GPR143 (XLR) \\
\hline Anterior segment dysgenesis & PITX3 (AD) \\
\hline Bietti crystalline corneoretinal dystrophy & $C Y P 4 V 2(A D)$ \\
\hline Blue cone monochromacy & OPN1LW; OPN1MW $(X L R)$ \\
\hline Brittle cornea syndrome & ZNF469; PRDM5 (AR) \\
\hline Cataract & BFSP2; CRYBA2; EPHA2 (AD) \\
\hline $\begin{array}{l}\text { Colobomatous macrophthalmia with } \\
\text { microcornea }\end{array}$ & $\mathrm{CH}$ \\
\hline Cone dystrophy & $K C N V 2(A D)$ \\
\hline Cone rod dystrophy & $\begin{array}{l}\text { C8orf37 (AR); RAB28 }(A R) ; R P G R(X L R) \\
\text { CACNA1F }(X L R)\end{array}$ \\
\hline Congenital microcoria & $\mathrm{CH}$ \\
\hline Congenital stationary night blindness & $\begin{array}{l}\text { NYX }(X L R) ; C A C N A 1 F(X L R) ; G R M 6(A R) ; \\
\text { SLC24A1 (AR); LRIT3 }(A R) ; G N B 3(A R) ; \\
\text { GPR179 (AR) }\end{array}$ \\
\hline Ectopia lentis et pupillae & ADAMTSL4 (AR) \\
\hline
\end{tabular}




\begin{tabular}{|c|c|}
\hline Ocular condition & Gene and inheritance pattern \\
\hline $\begin{array}{l}\text { High myopia with cataract and vitreoretinal } \\
\text { degeneration }\end{array}$ & $P 3 H 2(A R)$ \\
\hline Keratoconus & $V S X 1(A D)$ \\
\hline Leber congenital amaurosis & TULP1 (AR) \\
\hline $\begin{array}{l}\text { Microcornea, myopic chorioretinal atrophy, } \\
\text { and telecanthus }\end{array}$ & ADAMTS18 (AR) \\
\hline $\begin{array}{l}\text { Microspherophakia and/or megalocornea, } \\
\text { with ectopia lentis and/or secondary } \\
\text { glaucoma }\end{array}$ & $L T B P 2(A R)$ \\
\hline Ocular albinism & $O C A 2(A R)$ \\
\hline Primary open angle glaucoma & $M Y O C ; O P T N(A D)$ \\
\hline Retinal cone dystrophy & $K C N V 2(A R)$ \\
\hline Retinal dystrophy & C21orf2 (AR); TUB (AR) \\
\hline Retinitis pigmentosa & $\begin{array}{l}R P 1(A D) ; R P 2(X L R) ; R P G R(X L R) ; T T C 8 \\
(A R)\end{array}$ \\
\hline Sveinsson chorioretinal atrophy & TEAD1 $(A D)$ \\
\hline Vitreoretinopathy & ZNF408 (AD) \\
\hline Wagner vitreoretinopathy & $V C A N(A D)$ \\
\hline Weill-Marchesani syndrome & $\begin{array}{l}\text { ADAMTS10 }(A R) ; \text { FBN1 }(A D) ; L T B P 2(A R) ; \\
\text { ADAMTS17 }(A R)\end{array}$ \\
\hline
\end{tabular}

$A D$ autosomal dominant, $A R$ autosomal recessive, $X L R \mathrm{X}$ linked recessive, $C H$ chromosomal

pedigrees [21]. Families with genetic variants which show an autosomal dominant inheritance pattern were also most successful for myopia linkage studies. Up to now, 20 MYP loci [22-25] and several other loci [26-31] are identified for (high) myopia. Fine mapping led to candidate genes, such as the IGF1 gene located in the MYP3 locus [32]. Linkage using a complex inheritance design found five additional loci [33-37].

Validation of candidate genes often resulted in no association, but other variants appeared associated with the non-Mendelian, common form of myopia. This hints towards a genetic overlap between Mendelian and common myopia [38]. As the GWAS era progressed, linkage studies fell by the wayside. Nevertheless, segregation analyses combined with linkage and next generation sequencing (i.e. whole exome sequencing) of regions in pedigrees with high myopia are, in theory, expected to facilitate the discovery of rare variants with large effects; an aspect which cannot be distilled from GWAS.

\subsection{Candidate Gene Studies}

In candidate gene studies the focus is on a gene with suspected biological, physiological or functional relevance to myopia, in particular high myopia. Although sometimes effective, candidate gene studies are limited by their reliance on this existing knowledge. Table 5.2 summarizes candidate gene studies on (high) myopia. Particularly 


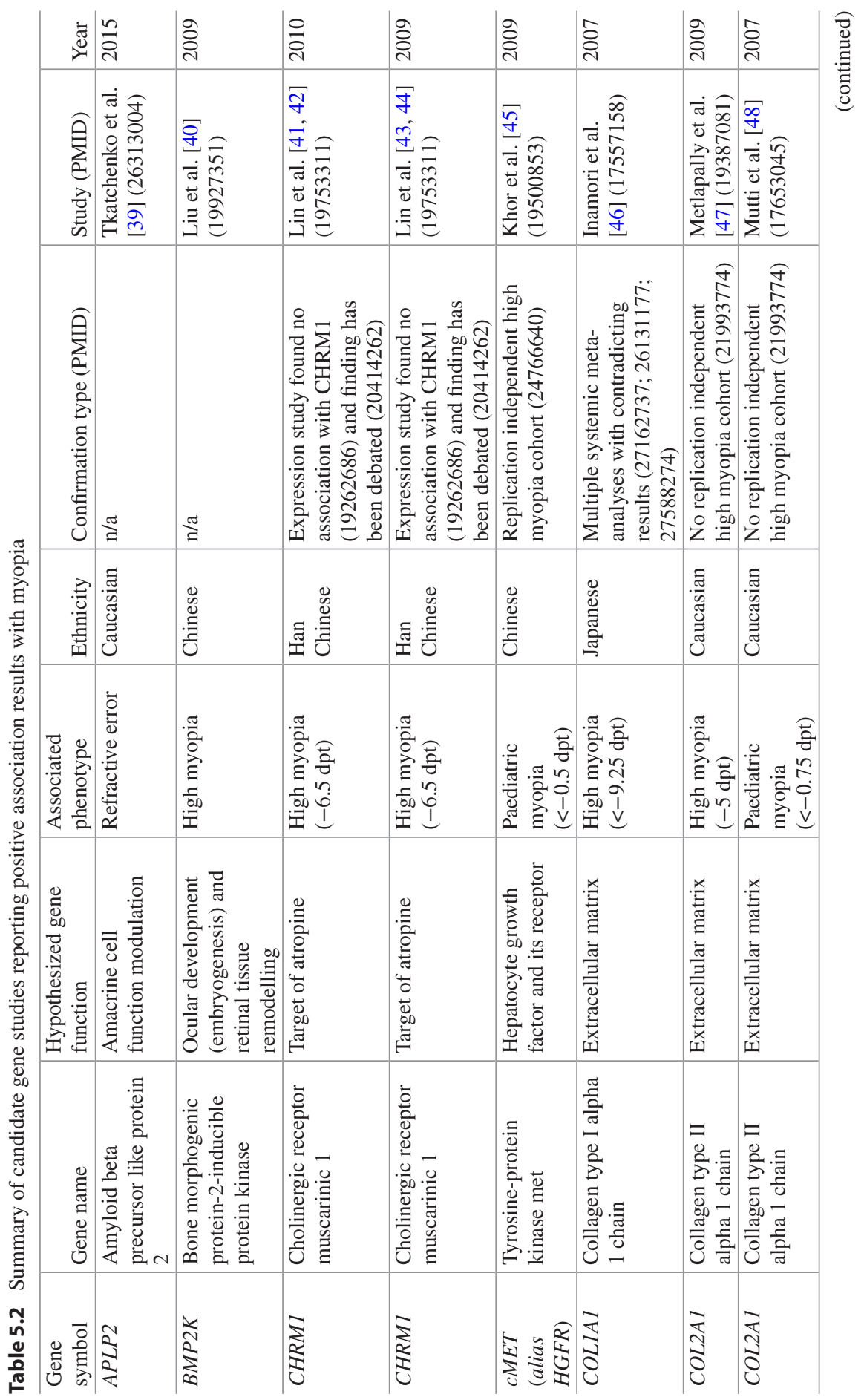




\begin{tabular}{|c|c|c|c|c|c|c|c|c|c|}
\hline & $\frac{\text { a }}{3}$ & |ః & 啇 & 总 & 윰 & $\overrightarrow{\vec{i}}$ & 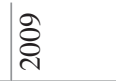 & |유. & 윰 \\
\hline 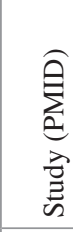 & 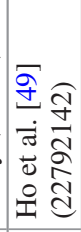 & 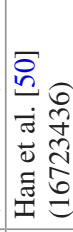 & 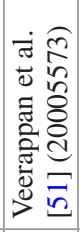 & 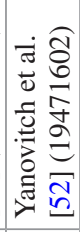 & 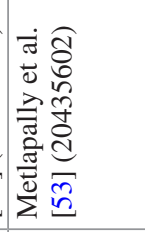 & 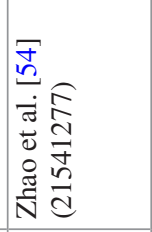 & 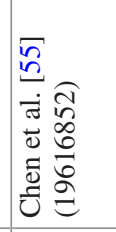 & 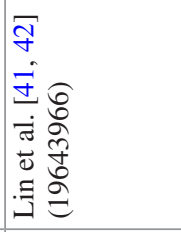 & 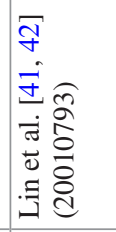 \\
\hline 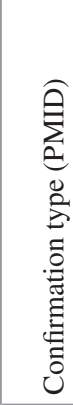 & 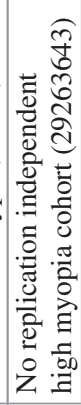 & 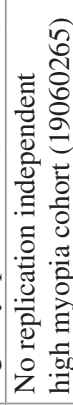 & 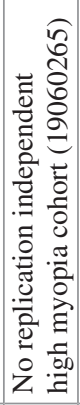 & 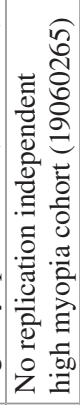 & 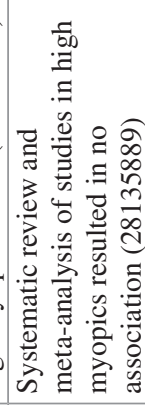 & 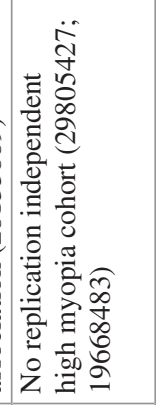 & 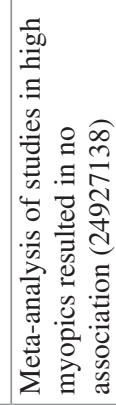 & 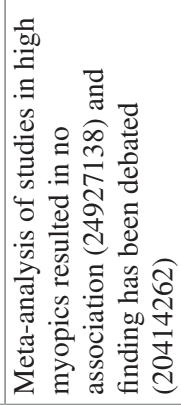 & 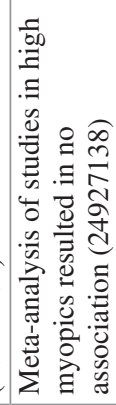 \\
\hline $\begin{array}{l}\stackrel{\overrightarrow{0}}{0} \\
\text { : } \\
\text { 岦 }\end{array}$ & 岁 & 离 & 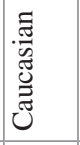 & 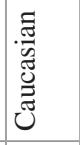 & 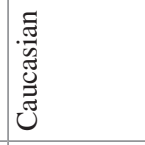 & 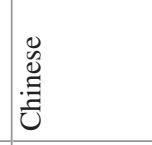 & 离 & 离 & 离 \\
\hline 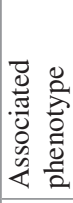 & 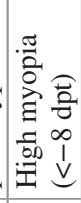 & 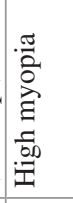 & 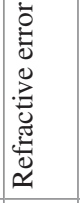 & 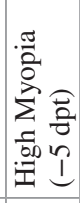 & 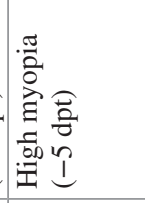 & 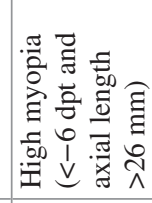 & 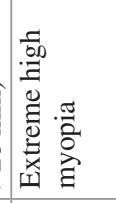 & 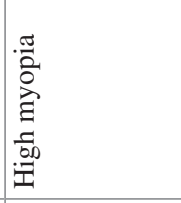 & 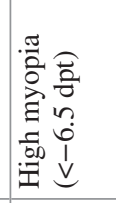 \\
\hline 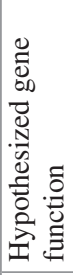 & 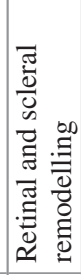 & 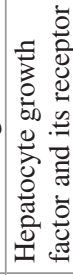 & 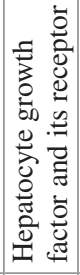 & 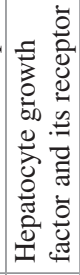 & 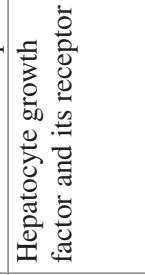 & 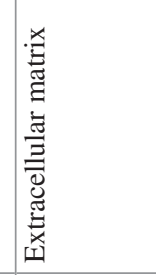 & 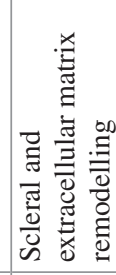 & 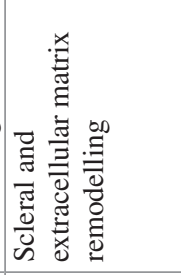 & 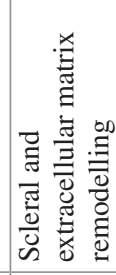 \\
\hline $\begin{array}{l}\stackrel{\Xi}{\Xi} \\
\stackrel{\Xi}{0} \\
\stackrel{0}{0} \\
0\end{array}$ & 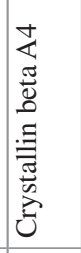 & 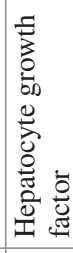 & 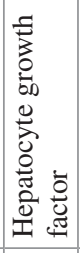 & 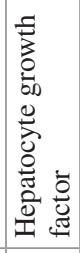 & 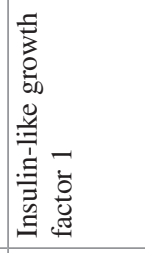 & 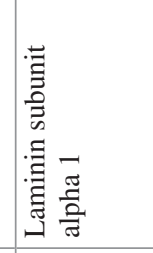 & 駕 & 氙 & 馬 \\
\hline 窇 & 辛 & ড্工 & S & ড & 选 & $\sum_{3}^{3}$ & 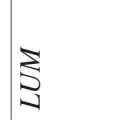 & 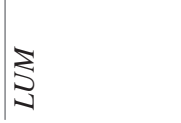 & $\underset{3}{\mathbb{3}}$ \\
\hline
\end{tabular}




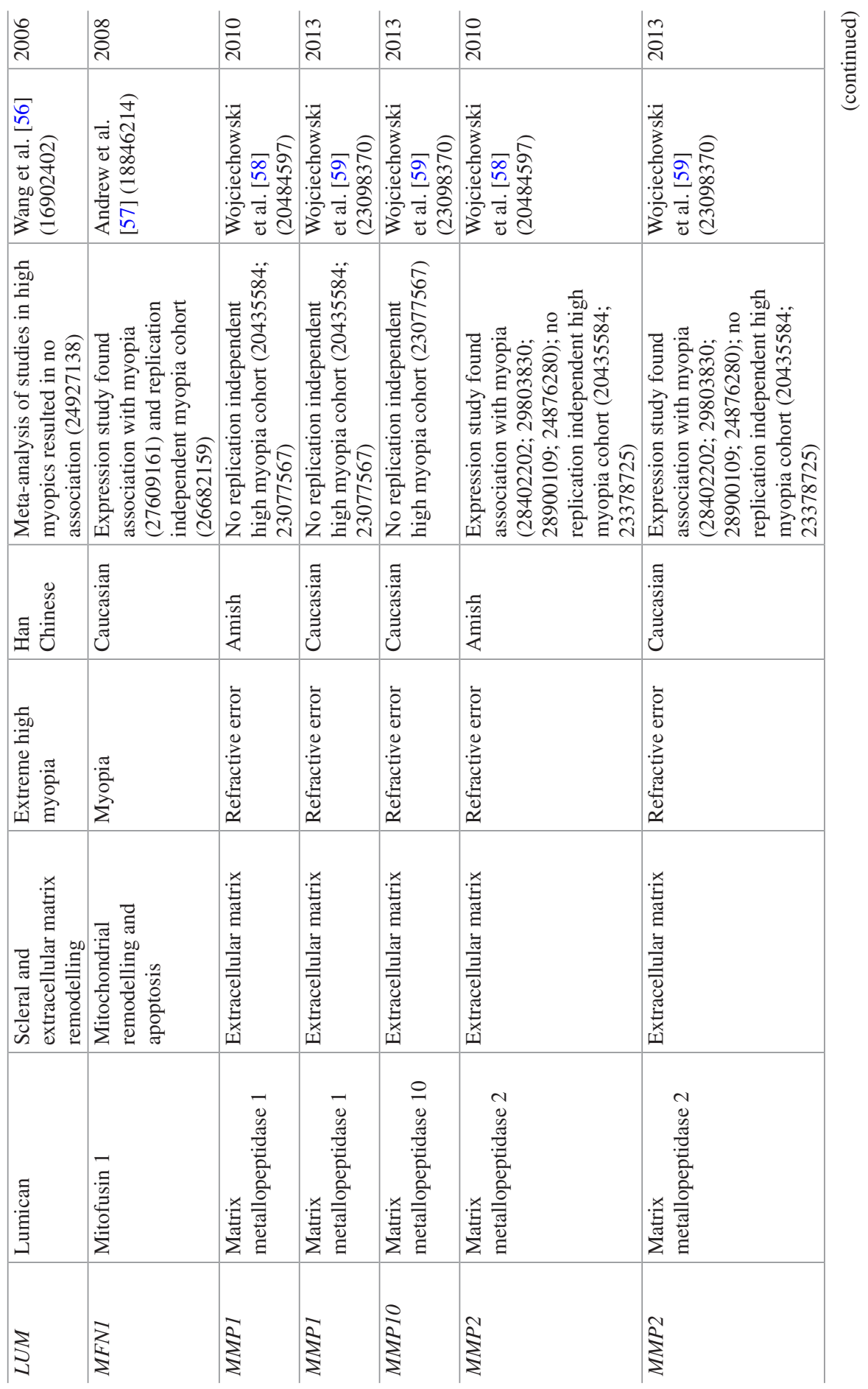




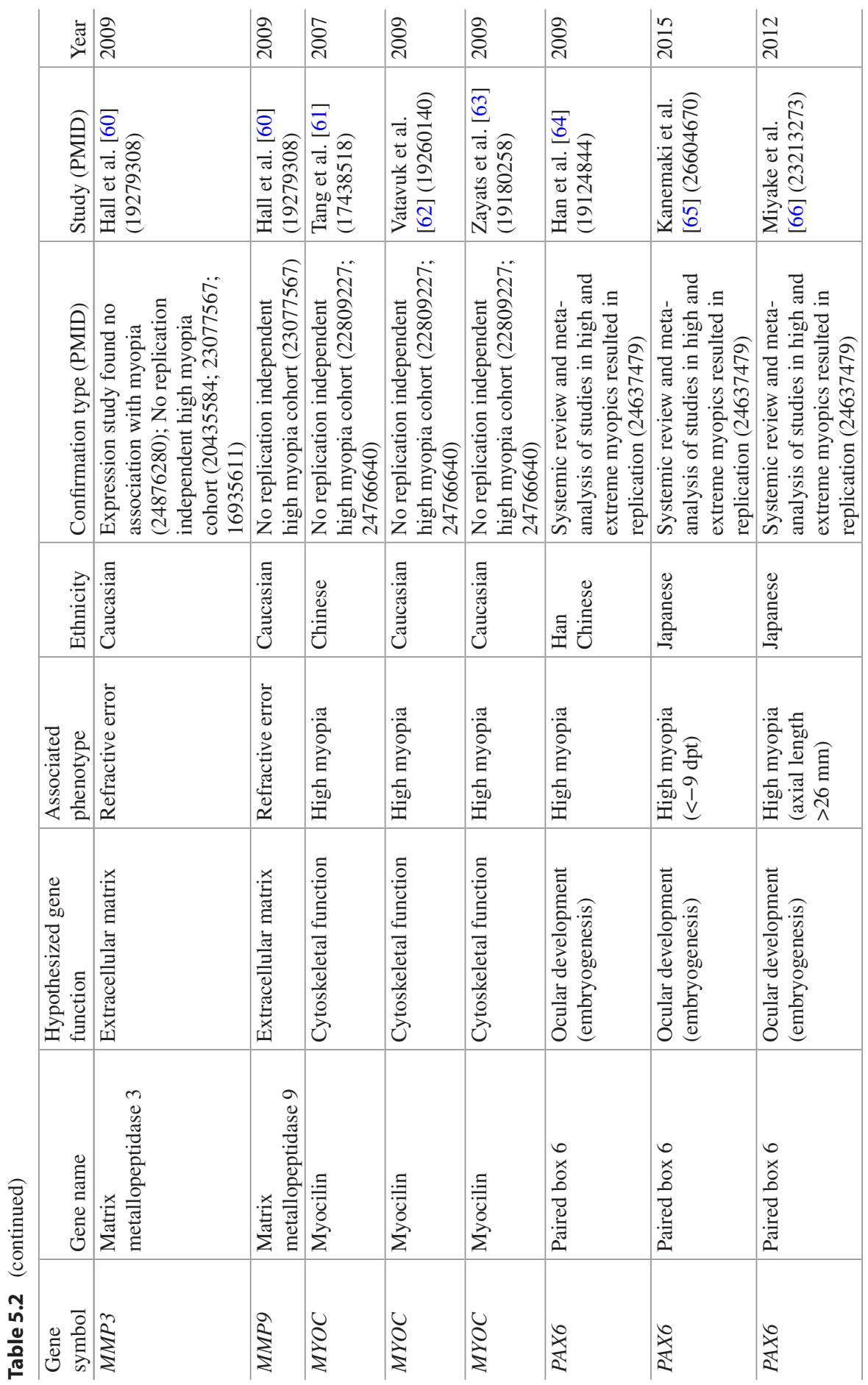




\begin{tabular}{|c|c|c|c|c|c|c|c|}
\hline ठ্ণ & 歩 & 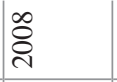 & 我 & 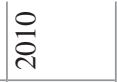 & ஜ̊ & $\frac{m}{\stackrel{n}{i}}$ & ठ্ণ \\
\hline 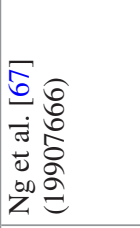 & 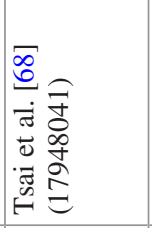 & 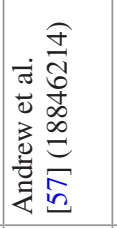 & 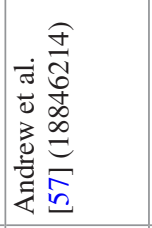 & 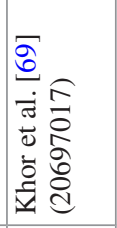 & 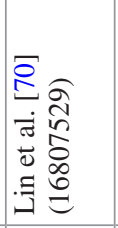 & 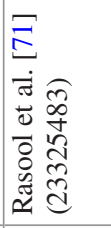 & 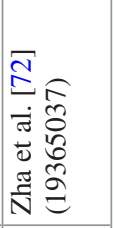 \\
\hline 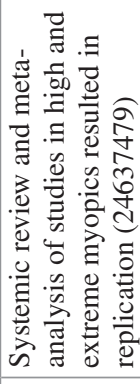 & 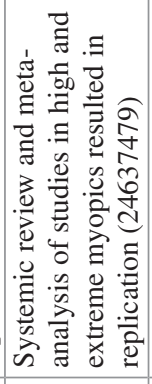 & $\frac{7}{2}$ & $\stackrel{\pi}{\beth}$ & 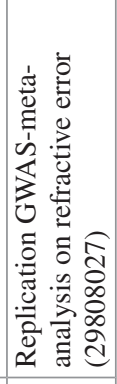 & 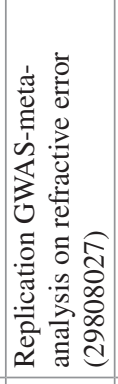 & 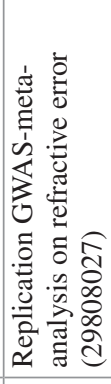 & 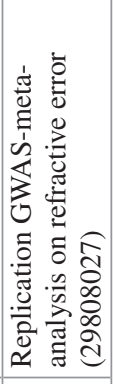 \\
\hline 胥 & 岁 & 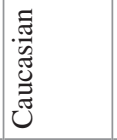 & 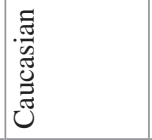 & 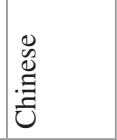 & 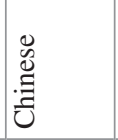 & : & 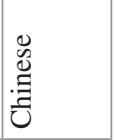 \\
\hline 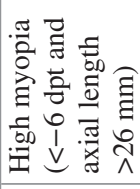 & 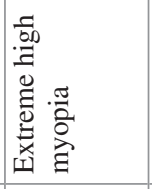 & 营 & 竞 & 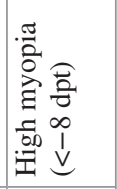 & 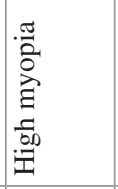 & 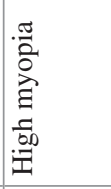 & 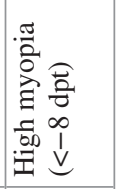 \\
\hline 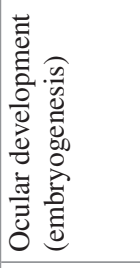 & 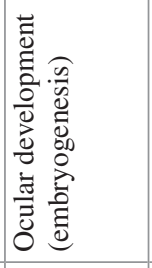 & 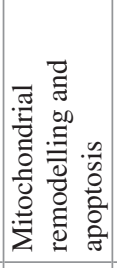 & 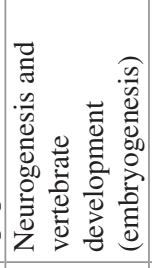 & 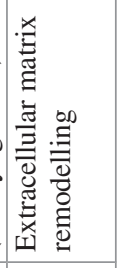 & 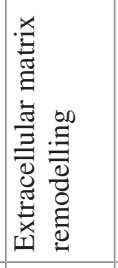 & 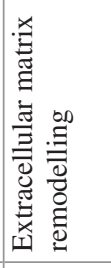 & 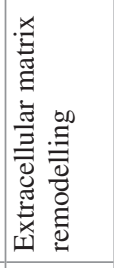 \\
\hline 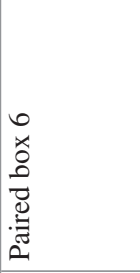 & 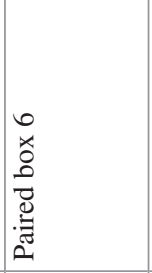 & 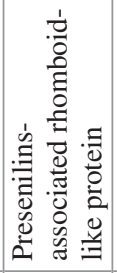 & 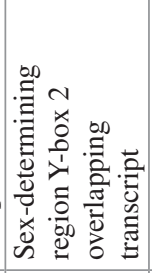 & 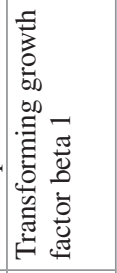 & 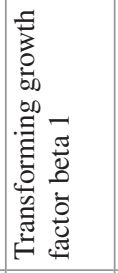 & 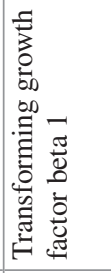 & 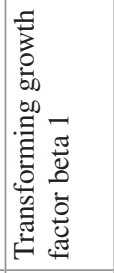 \\
\hline$\frac{1}{2}$ & $\underset{2}{2}$ & $\begin{array}{l}\overrightarrow{2} \\
\frac{2}{2} \\
2\end{array}$ & 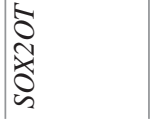 & 造 & 造 & 造 & 造 \\
\hline
\end{tabular}




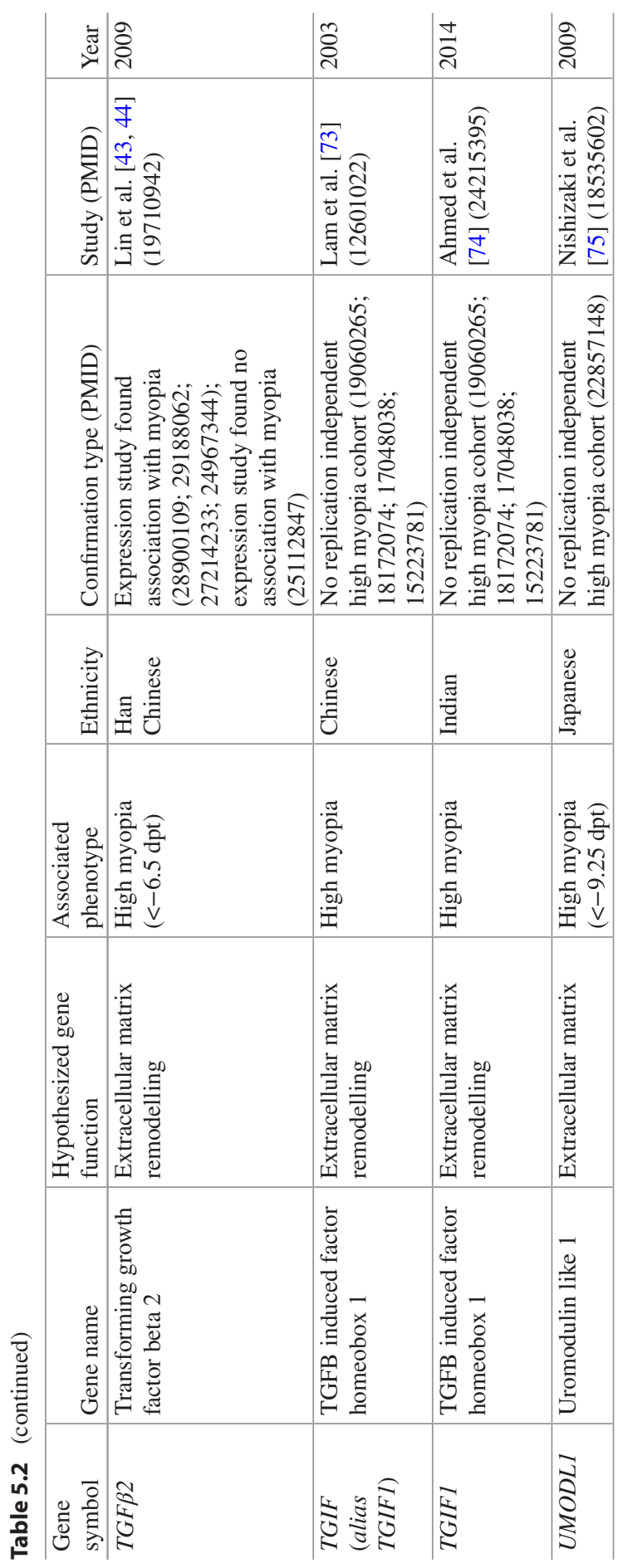


notable are genes encoding extracellular matrix-related proteins (COL1A1, COL2A1 $[16,17]$ and $M M P 1, M M P 2, M M P 3, M M P 9, M M P 10[59,60])$. For candidates such as $P A X 6$ and $T G F B 1$, the results were replicated in multiple independent extreme/high myopia studies and validated in a large GWAS meta-analysis in 2018, respectively $[18,76]$. However in most other cases, the results were not independently validated: $L U M$ and $I G F 1$ failed to confirm an association [77, 78]. Interestingly, in a few cases the candidates were subsequently implicated in GWAS of other ocular traits: TGF 32 and $L U M$ for central corneal thickness (CCT), a glaucoma and keratoconus endophenotype [14], PAX6 with optic disc area [79] and $H G F$ [80].

\subsection{Genome-Wide Association Studies}

Generally, linkage studies are limited to identification of genetic variants with a large effect on myopia [81]. Given the limited number of genes identified by linkage, it became apparent in the 2000s that identifying large numbers of additional myopia genes was more practical with genome-wide association studies (GWASes), since it has dramatically higher statistical power. GWASes have greatly enhanced our knowledge of the genetic architecture of (complex) diseases [82]. Most of the variants found via GWAS reside in non-exonic regions and their effect sizes are typically small [82, 83]. For GWAS, $200 \mathrm{k}-500 \mathrm{k}$ genetic markers are usually genotyped and a further $>10$ million "imputed", taking advantage of the correlation structure of the genome. This approach is most effective for common variants (allele frequencies $>0.01$ in the population, although with larger reference panels, rarer alleles can also be detected).

Initially, GWASes for myopia were performed as a dichotomous outcome (i.e. case-control, Table 5.3). Since myopia constitutes a dichotomization of the quantitative trait spherical equivalent, considering the quantitative trait should be more informative for gene mapping. The first GWASes for spherical equivalent were conducted in 2010 [96, 97], with 4000 individuals required to identify the first loci. The first loci to reach the genome-wide significance threshold $\left(P<5 \times 10^{-8}\right.$, the threshold reflecting the large number of statistical tests conducted genome-wide) were markers near the RASGFRl gene on $15 \mathrm{q} 25.1\left(P=2.70 \times 10^{-9}\right)$ and markers near GJD2 on $15 q 14\left(P=2.21 \times 10^{-14}\right)$. A subsequent analysis combining five cohorts $(N=7000)$ identified another locus at the RBFOXI gene on chromosome $16\left(P=3.9 \times 10^{-9}\right)$ [98].

These early efforts made it clear that individual groups would have difficulty in mapping many genes for spherical equivalent, motivating the formation of the Consortium for Refractive Error and Myopia (CREAM) in 2010, which included researchers and cohorts from the USA, Europe, Asia and Australia. They replicated SNPs in the 15q14 loci [99], which was further affirmed by other studies on both spherical equivalent and axial length alongside with the replication of the $15 q 25$ locus $[100,101]$.

In 2013, two major GWAS meta-analyses on refractive error traits (spherical equivalent and age of spectacle wear) identified 37 novel loci (Table 5.4), with robust replication of GJD2, RFBOX1 and RASGFR1 in both meta-analyses. The first was the collaborative work of CREAM based on a GWAS meta-analysis on spherical equivalent, comprising 35 individual cohorts $\left(N_{\text {European }}=37,382\right.$; $N_{\text {SoutheastAsian }}=12,332$ ) [108]. 23andMe, a direct-to-consumer genetic testing company, performed the second major GWAS, replicating 8 of the novel loci found by CREAM and identifying another 11 novel loci based on a GWAS survival analysis 


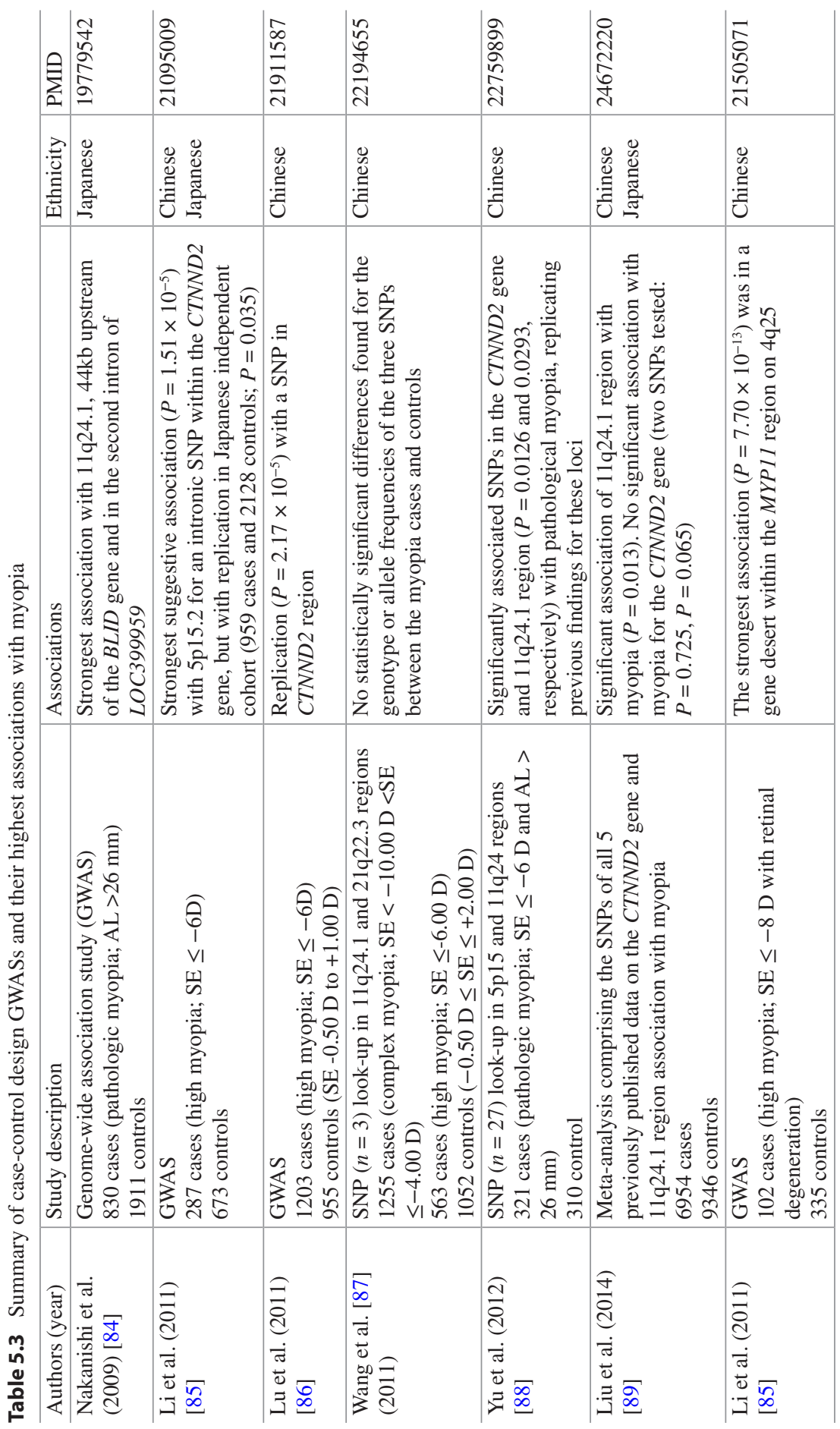




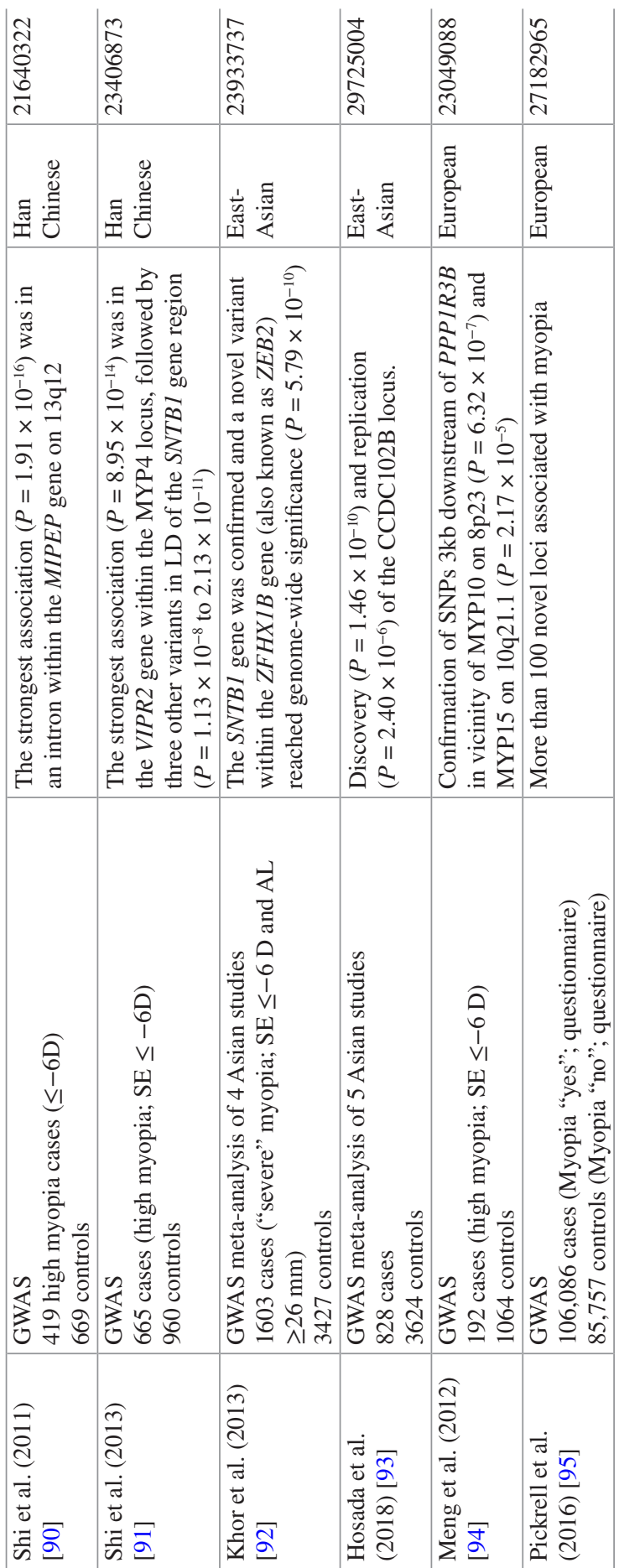




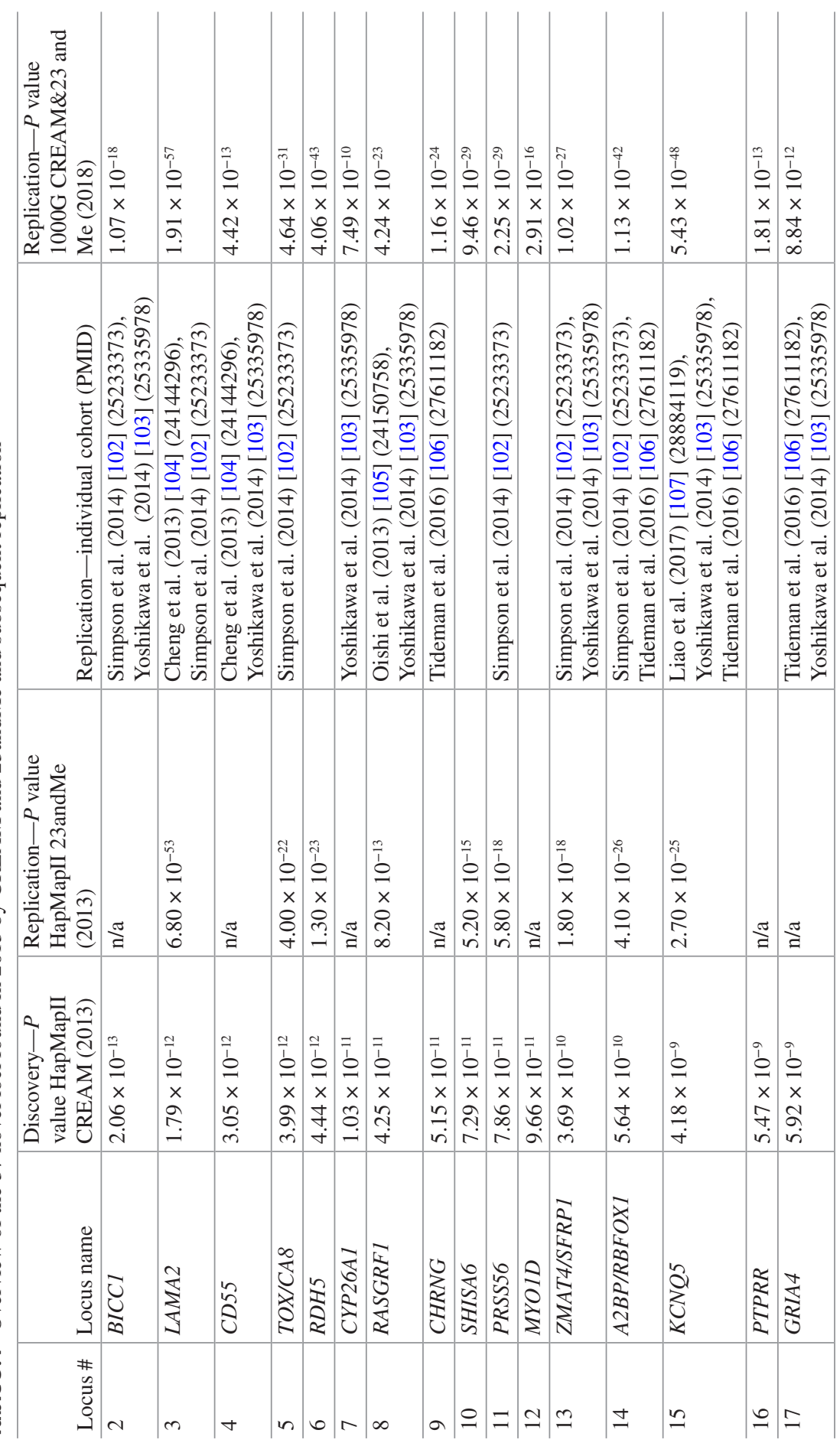




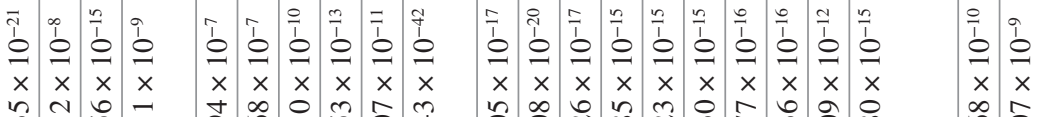

m

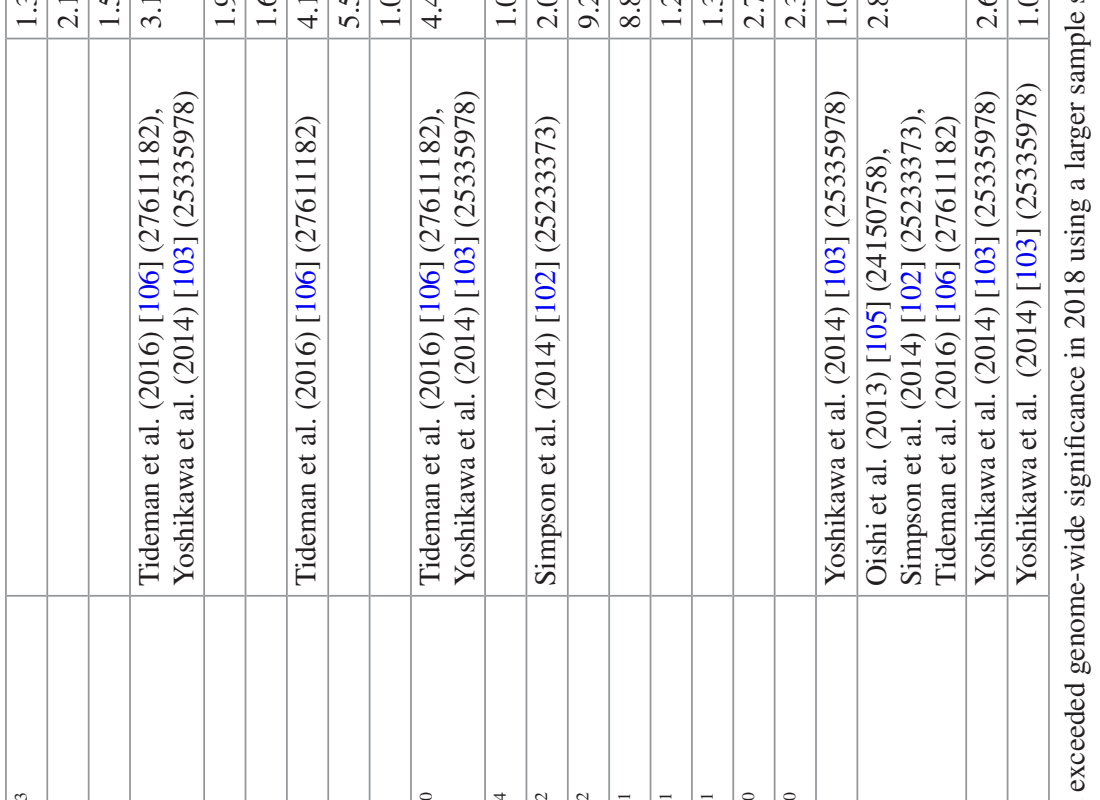

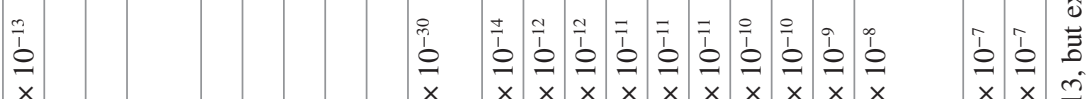

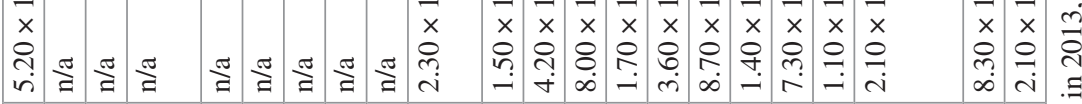

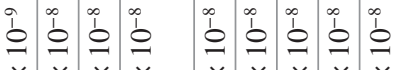

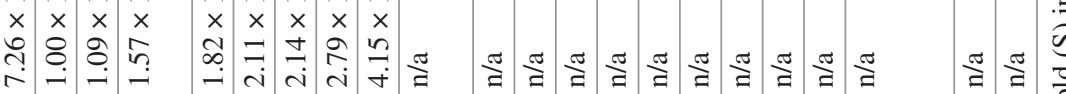

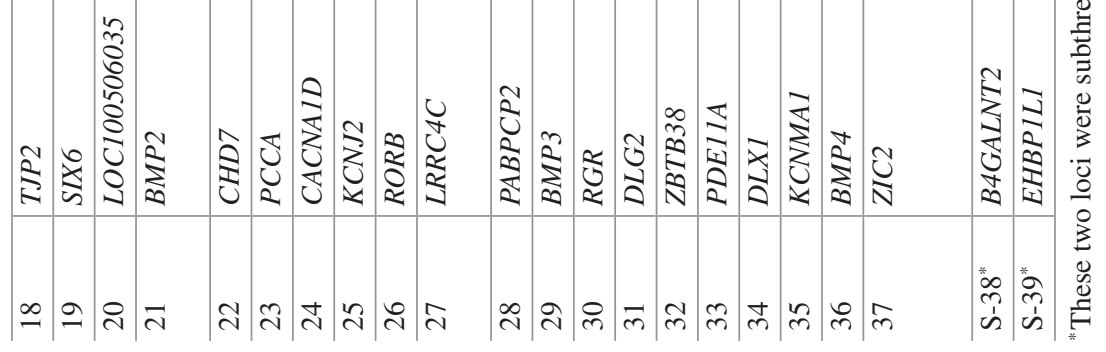

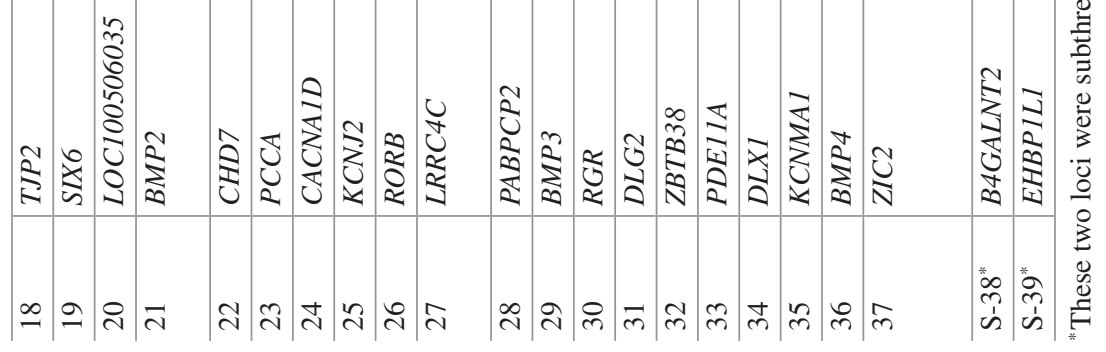

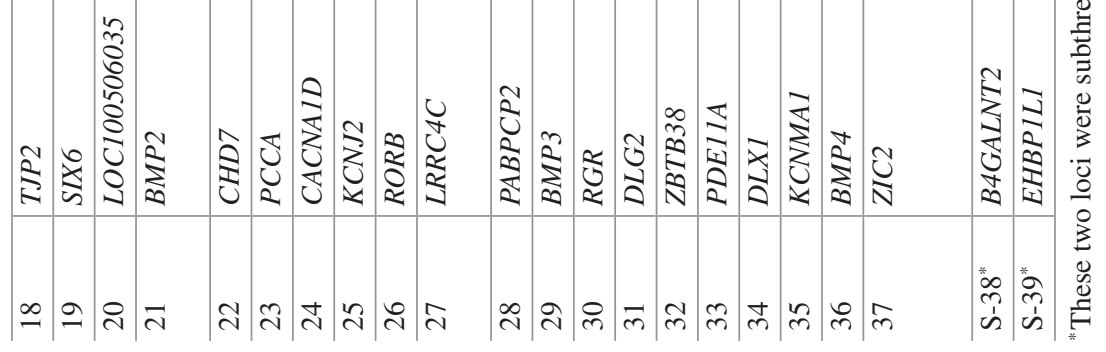

离 $\stackrel{\Xi}{\Xi}$

$\Xi$

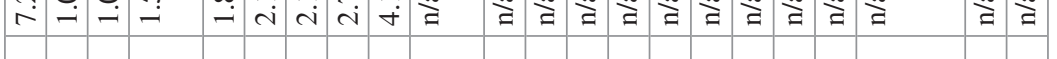


of age of spectacle wear in 55,177 participants of European ancestry. To the surprise of some in the field, the effect sizes and direction of the effects of the loci found by these two groups were concordant despite the difference in phenotype definition and in scale: dioptres for CREAM and hazard ratios for 23andMe [109]. Subsequently, replication studies provided validation for the associated loci and highlighted two other suggestive associations. At this point, the implicated loci explained $3 \%$ of the phenotypic variance in refractive error $[108,110]$.

The CREAM and 23andMe studies represented a large increase in sample size over the initial GWASs. Their meta-analysis approach was very effective in discovering new loci. This motivated joined efforts of CREAM and 23andMe, which resulted in a GWAS meta-analysis including 160,420 participants. Moreover, a denser imputation reference set was used (1000G phase 1 version 3 ), enabling better characterization of genetic variations. Although CREAM and 23andMe used different phenotypes (spherical equivalent and age at first spectacle wear, respectively) again the results were concordant and the new findings were replicated in an independent cohort with refractive error available (UK biobank, comprising 95,505 participants). Overall, this GWAS increased the number of risk loci to 161, explaining $7.8 \%$ of the phenotypic variance in refractive error. Very large sample sizes (millions) will be required to identify all of the loci contributing to myopia risk.

The genetic correlation was estimated to be 0.78 between European and Asian ancestry, suggesting that despite (1) large differences in the rate of myopia between these groups and (2) differences in the genetic ancestry of these groups, most of the genetic variation is in common. Figure 5.1 provides the chronological discovery of all associated loci and Fig. 5.2 shows the effect sizes of the established 161 loci.

Several "endophenotypes" have been considered for myopia: spherical equivalent, axial length, corneal curvature and age of diagnosis of myopia. Axial length is a wellstudied "endophenotype" which correlates strongly with refractive error. The first GWAS of axial length considered 4944 Asian ancestry individuals and identified a locus at 1q41. A subsequent meta-analysis combining data on 12,531 European and 8216 Asian ancestry individuals uncovered a further eight genome-wide significant loci at RSPO1, C3orf26, LAMA2, GJD2, ZNRF3, CD55, MIP, ALPPL2, as well as confirming the 1q41 locus. Five of the axial length loci were also associated loci for refractive error. GWASs performed for corneal curvature [104, 111-114] identified the loci FRAP1, CMPK1, $R B P 3$ and PDGFRA; in the case of PDGFRA, associations have also been found with eye size. A study in 9804 Japanese individuals and replication in Chinese and European ancestry cohorts analysed three myopia-related traits (refractive error, axial length and corneal curvature). They replicated the association of GJD2 and refractive error as well as the association of SNPs in $W N T 7 B$ for axial length and corneal curvature $[114,115]$

\subsection{Pathway Analysis Approaches}

GWAS approaches improve our understanding of the molecular basis of traits by mapping individual loci. However, it is possible to place such loci into a broader context by applying pathway analysis approaches. In myopia, a retina-to-sclera signaling cascade has been postulated, but the specific molecular components were unclear. Recent GWASs have uncovered genes which lie along this pathway [108, 110, 116] — genetic changes at individual loci only make small changes to phenotype but collectively these 


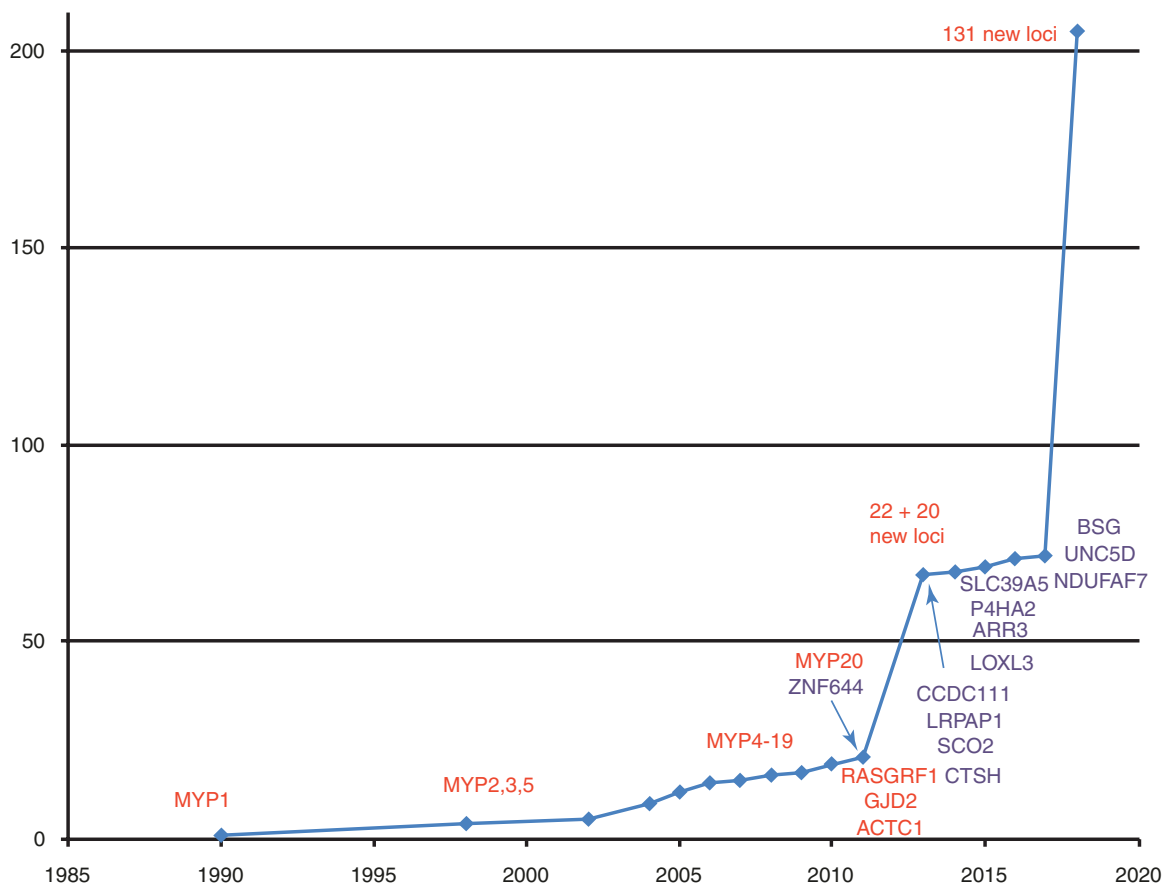

Fig. 5.1 Historic overview of myopia gene finding. Overview of myopia gene finding in historic perspective. Genes identified using whole exome sequencing are marked as purple. Other loci (linkage studies, GWAS) are marked as red

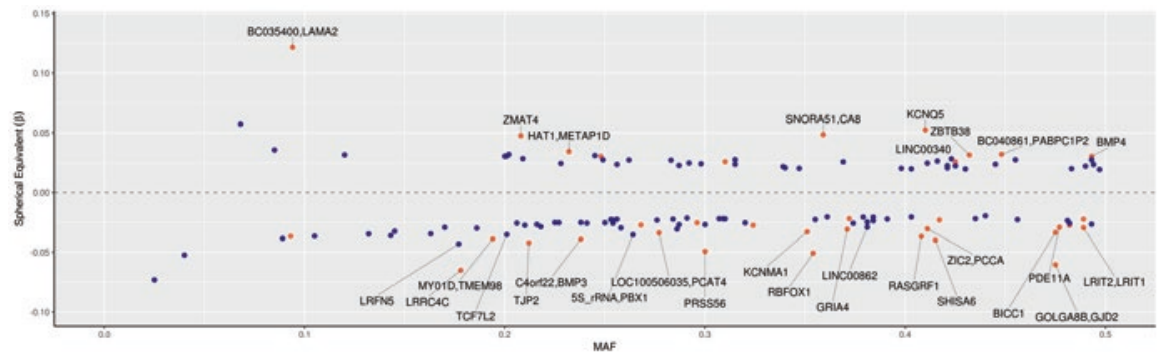

Fig. 5.2 Effect sizes of common and rare variants for myopia and refractive error. Overview of SNPs and annotated genes found in the most recent GWAS meta-analysis [18]. X-axis displays the minor allele frequency of each SNP; y-axis displays the effect size of per individual SNP. The blue dots represent the novel loci discovered by Tedja et al. [18] and the pink dots represent the loci found by Verhoeven et al. [108], which now have been replicated

perturbations are responsible for larger changes in the retina-to-sclera signaling cascade, ultimately explaining differences in refractive error from individual to individual.

Pathways inferred from the first large-scale CREAM GWAS [108, 110] included neurotransmission (GRIA4), ion transport (KCNQ5), retinoic acid metabolism (RDH5), extracellular matrix remodelling (LAMA2, BMP2) and eye development (SIX6, PRSS56). The 23andMe GWAS identified an overlapping set of pathways: neuronal development (KCNMA1, RBFOX1, LRRC4C, NGL-1, DLG2, TJP2), 


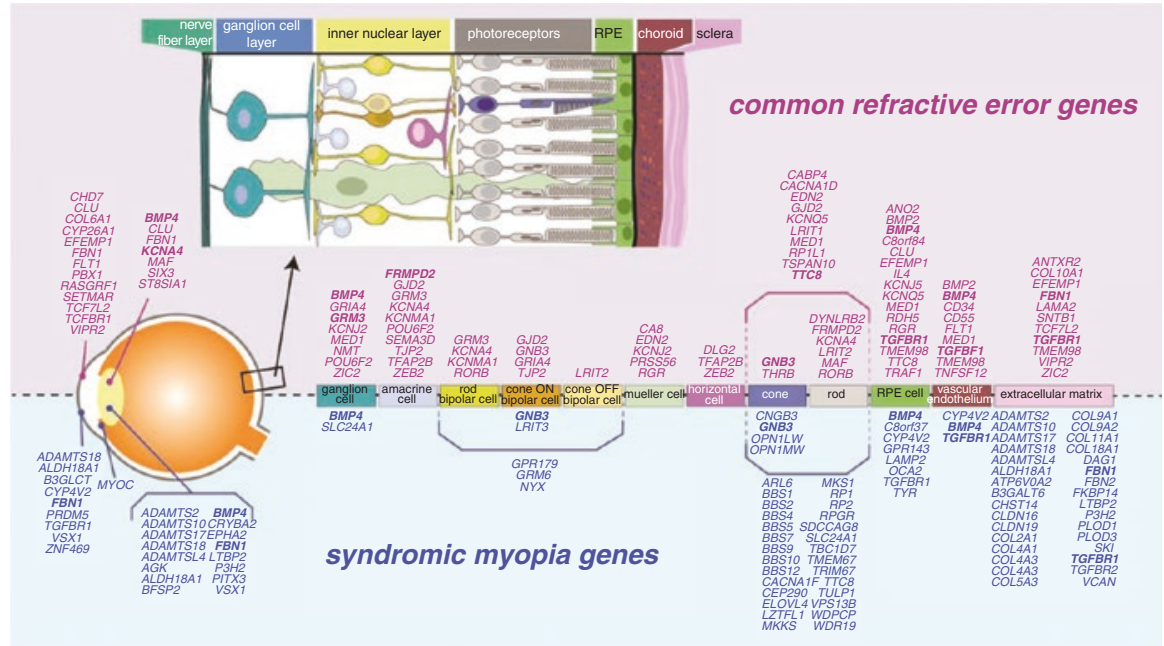

Fig. 5.3 Schematic overview of known function in retinal cell types of refractive error and syndromic myopia genes according to literature. Bold: genes identified for both common refractive error and in syndromic myopia

extracellular matrix remodelling (ANTXR2, LAMA2), the visual cycle ( $R D H 5, R G R$, $K C N Q 5)$, eye and body growth (PRSS56, BMP4, ZBTB38, DLX1) and retinal ganglion cell (ZIC2, SFRP1) [117]. When considered in the context of known proteinprotein interactions, many genes in these pathways are related to growth and cell cycle pathways, such as the TGF-beta/SMAD and MAPK pathways [118].

The most recent meta-analysis combining data from CREAM and 23andMe data taken together confirmed previous findings and offered additional insights [18]. In a gene-set analysis, several pathways were highlighted including "abnormal photoreceptor inner segment morphology" (Mammalian Phenotype Ontology (MP) 0003730); "thin retinal outer nuclear layer" (MP 0008515); "nonmotile primary cilium" (Gene Ontology (GO) 0031513); "abnormal anterior-eye-segment morphology" (MP 0005193) and "detection of light stimulus" (GO 0009583). The genes implicated in this large-scale GWAS were distributed across all cell types in the retina-to-sclera signaling cascade (neurosensory retina, RPE, vascular endothelium and extracellular matrix, Fig. 5.3). The larger GWAS also suggested novel mechanisms, including angiogenesis, rod-and-cone bipolar synaptic neurotransmission and anterior-segment morphology. Interestingly, a novel association was found at the DRDI gene, supporting previous work linking the dopamine pathway to myopia.

\subsection{Next Generation Sequencing}

GWAS approaches have been highly effective in assessing the role of common variants in myopia but such methods cannot effectively characterize very rare genomic variants. Whole exome sequencing (WES) allows investigation of rare variants in exonic regions; due to cost, applications to date have primarily been in family studies or studies of early onset high myopia. 
Studies employing WES to date have either focused on family designs (e.g. particular inheritance patterns such as X-linkage or conditions such as myopic anisometropia) or case-control studies of early onset high myopia [119-122]. The WES-based approaches identified several novel mutations in known myopia genes (Table 5.5). For instance, Kloss et al. [131] performed WES on 14 families with high myopia, identifying 104 genetic variants in both known MYP loci (e.g. AGRN, EME1 and HOXA2) and in new loci (e.g. ATL3 and AKAP12) [131]. In the family studies, most variants displayed an autosomal dominant mode of inheritance $[119,123,124,130]$ although X-linked heterozygous mutations were found in ARR3 [126].

Both retinal dystrophies and ocular development disorders coincide with myopia. Sun et al. [132] investigated if there was a genetic link by evaluating a large number of retinal dystrophy genes in early onset high myopia. They examined 298 unrelated myopia probands and their families, identifying 29 potentially pathogenic mutations in COL2A1, COL11A1, PRPH2, FBN1, GNAT1, OPA1, PAX2, GUCY2D, TSPAN12, CACNAIF and RPGR with mainly an autosomal dominant pattern.

\subsection{Environmental Influences Through Genetics}

Although myopia is highly heritable within specific cohorts, dramatic changes in environment across many human populations have led to large changes in prevalence over time [133-136]. The role of changes in socioeconomic status, time spent outdoors, education and near-work are now well established as risk factors for myopia, based on observational studies [137-139]. Education has proven the most influential and consistent factor, with a doubling in myopia prevalence when attending higher education compared to finishing only primary education [140-142]. There are two main areas where genetic studies can inform our understanding of the role of environment. Firstly, gene-environment studies can highlight where interactions exist. Secondly, observational studies only establish association and not causation-in some circumstances genetic data can be used to strengthen the case for an environmental risk factor causally (or not) influencing myopia risk (Mendelian randomization).

Gene-environment (GxE) interaction analyses examine whether genes operate differently across varying environments. GxE studies in myopia have focused primarily on education. An early study in North American samples examined GxE for myopia and the matrix metalloproteinases genes (MMP1-MMP10): a subset of SNPs were only associated with refraction in the lower education level [58, 59]. A subsequent study in five Singapore cohorts found variants in DNAH9, GJD2 and ZMAT4, which had a larger effect on myopia in a high education subset [143]. Subsequent efforts to examine GxE considered the aggregate effects of many SNPs together. A study in Europeans found that a genetic risk score comprising 26 genetic variants was most strongly associated with myopia in individuals with a university level education [144]. A study examining GxE in children considered near work and time outdoors in association with 39 SNPs and found weak evidence for an interaction with near work [144, 145]. Finally, a CREAM study was able to identify additional myopia risk loci by allowing for a GxE approach [19].

Mendelian randomization (MR) infers whether a risk factor is causally associated with a disease. MR exploits the fact that germline genotypes are randomly 


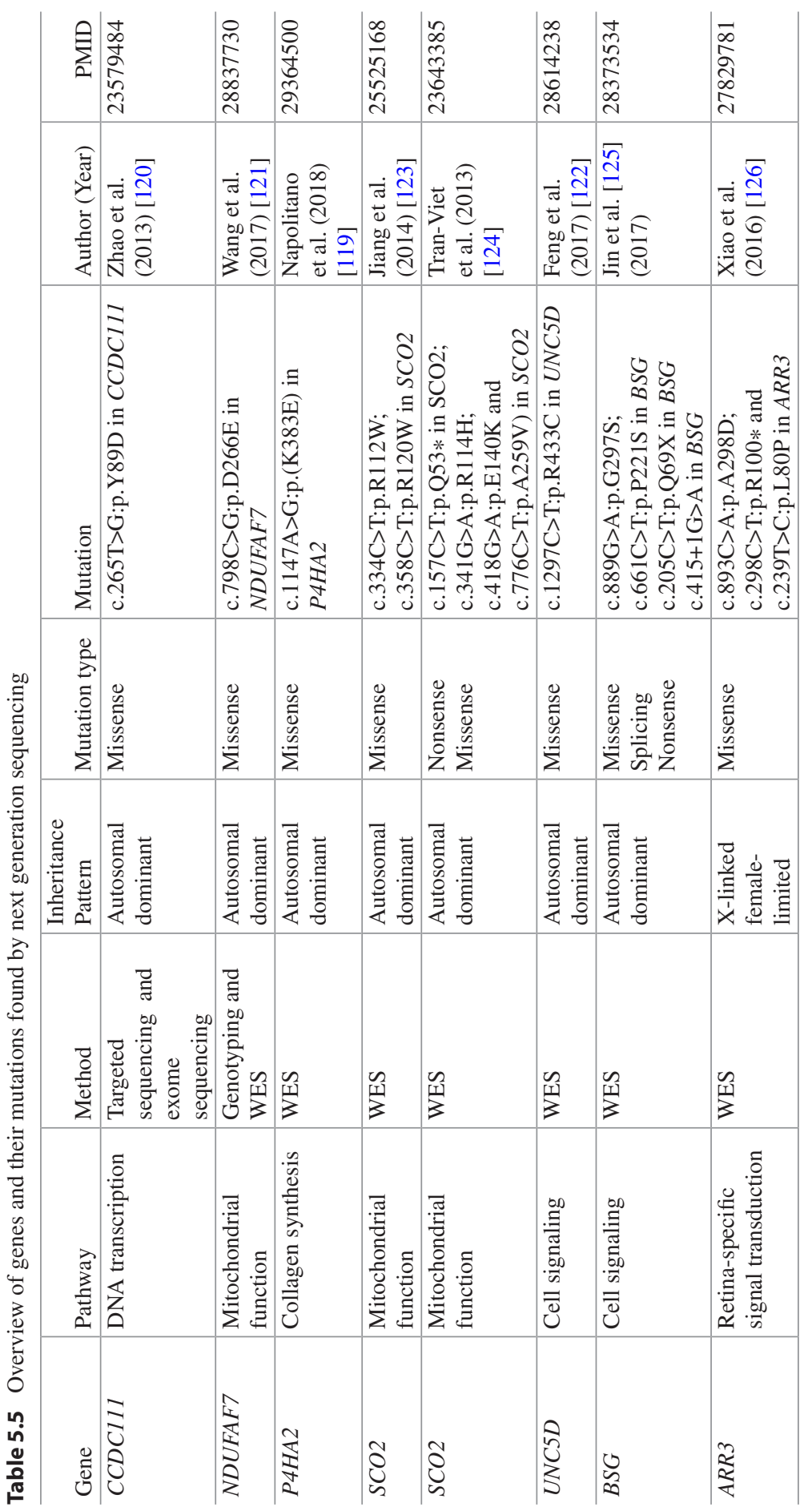




\begin{tabular}{|c|c|c|c|c|c|c|c|c|}
\hline 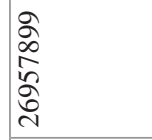 & 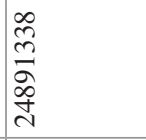 & $\begin{array}{l}\infty \\
0 \\
n \\
n \\
n \\
n \\
n\end{array}$ & $\mid \begin{array}{l}\nexists \\
\bar{n} \\
\infty \\
\infty \\
\infty \\
\end{array}$ & $\mid \begin{array}{l}\infty \\
0 \\
\sim \\
\tilde{n} \\
\tilde{n} \\
\end{array}$ & 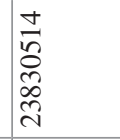 & $\begin{array}{l}\overline{\tilde{a}} \\
\tilde{a} \\
\hat{\sigma} \\
\text { ते }\end{array}$ & $\begin{array}{l}\infty \\
0 \\
n \\
n \\
n \\
n \\
n\end{array}$ & \begin{tabular}{l}
9 \\
\multirow{2}{*}{} \\
$\infty$ \\
$\infty$ \\
$\infty$ \\
$N$
\end{tabular} \\
\hline 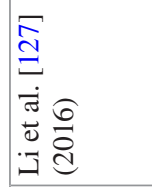 & 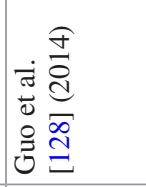 & 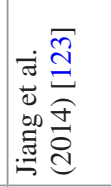 & 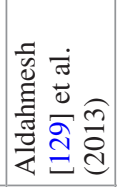 & 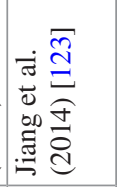 & 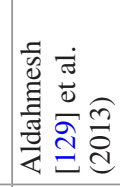 & 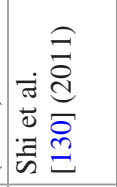 & 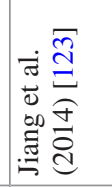 & 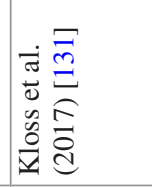 \\
\hline 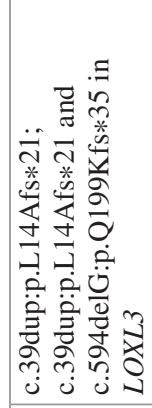 & 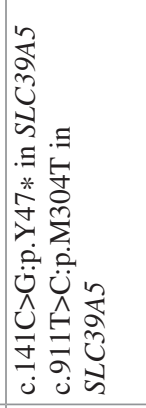 & 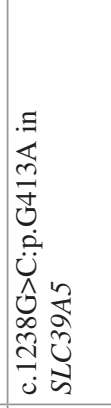 & 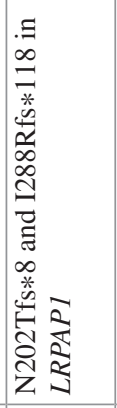 & 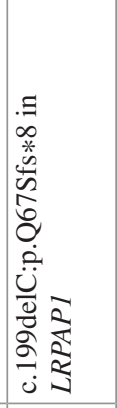 & 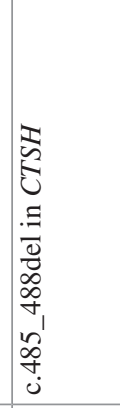 & 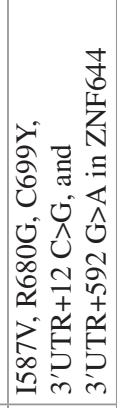 & 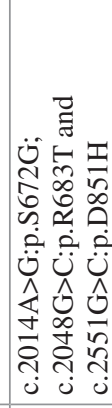 & 1 \\
\hline 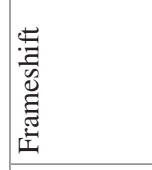 & 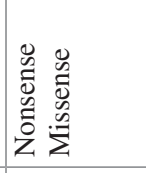 & 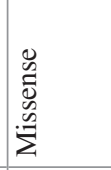 & 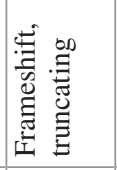 & 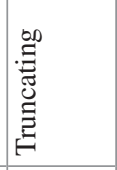 & 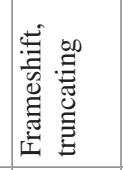 & 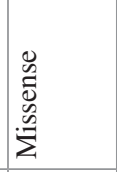 & 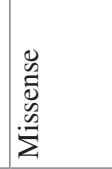 & z \\
\hline 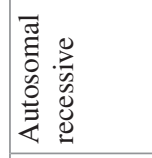 & 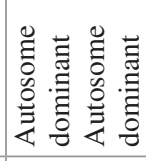 & 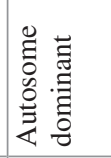 & 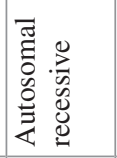 & 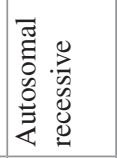 & 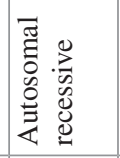 & 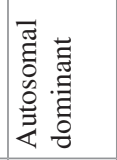 & 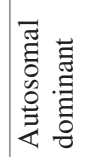 & 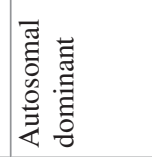 \\
\hline$\frac{5}{3}$ & $\sum_{3}^{\infty}$ & $\sum_{1}^{2}$ & $\begin{array}{l}n \\
11 \\
3\end{array}$ & $\frac{1}{11}$ & $\sum_{3}^{2}$ & $\frac{2}{1}$ & $\stackrel{n}{1}$ & 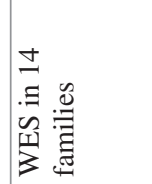 \\
\hline 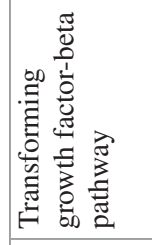 & 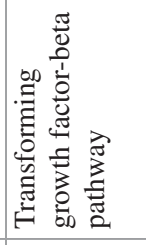 & 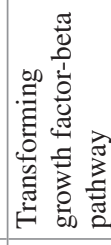 & 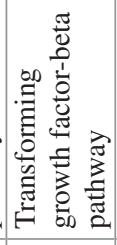 & 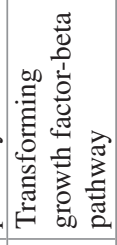 & 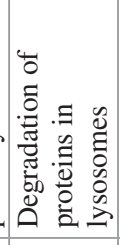 & 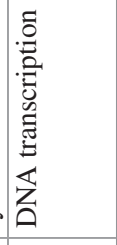 & 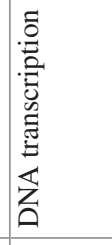 & 1 \\
\hline 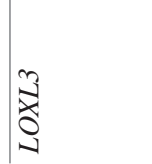 & 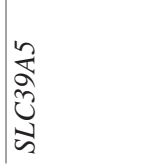 & 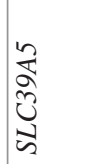 & $\frac{\substack{d \\
\frac{2}{y}}}{2}$ & 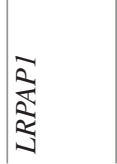 & ర్ & \begin{tabular}{l}
$\mathbf{J}$ \\
L \\
\multirow{2}{*}{}
\end{tabular} & 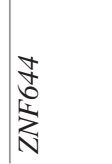 & 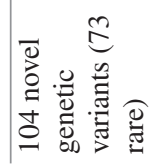 \\
\hline
\end{tabular}


assigned at meiosis, to enable a "natural" randomized controlled trial. Since the assigned genotypes are independent of non-genetic confounding and are unmodified by disease processes, MR offers a better assessment of causality than that available from observational studies [146, 147].

Two MR studies found a causal effect of education on the development of myopia. One of the MR studies tested for causality bi-directionally [148]. Both found a larger effect through MR than that estimated from observational studies suggesting that confounding in observational studies may have been obscuring the true relationship [149]. As expected, there was little evidence of myopia affecting education ( -0.008 years/ dioptre, $P=0.6$ ). Another study focused on the causality of low vitamin $\mathrm{D}$ on myopia due to controversy in the literature [150]. The estimated effects of vitamin D on refractive error were so small (Caucasians: -0.02 [95\% CI -0.09, 0.04] dioptres (D) per $10 \mathrm{nmol} / \mathrm{l}$ increase in vitamin D concentration; Asians: 0.01 [95\% CI -0.17, 0.19] D per $10 \mathrm{nmol} / \mathrm{l}$ increase) that the authors concluded that the true contribution of vitamin D levels to degree of myopia is probably zero and that previous observational findings were likely confounded by the effects of time spent outdoors.

\subsection{Epigenetics}

Epigenetics in refractive error and myopia is postulated to be important due to the known effects of environmental factors on refractive error and myopia development. Nevertheless, this field is still developing and some characteristics of epigenetics render it a difficult issue to unravel. Epigenetic features can be influenced by environmental factors and are time dependent and tissue specific. This complicates the study of these effects, since myopia and refractive errors develop during childhood and young adolescence and obtaining eye tissue, preferably retinal and scleral would be unethical. Furthermore, although some epigenetic processes are conserved across species, this is not always the case: making animal studies not always translational to humans.

Non-Coding RNAs and Myopia The latest GWAS meta-analysis found 31 of 161 loci residing in or near regions transcribing (small) noncoding RNAs, thus hinting towards the importance of epigenetics $[18,151]$. MicroRNAs, or miRNAs, are the best-characterized family of small non-coding RNAs. They are approximately 19-24 nucleotides in length in their mature form. They are able to bind to 3' UTR regions on RNA polymers by sequence-specific post-transcriptional gene silencing; one miRNA can regulate the translation of many genes. MiRNAs have been a hot topic in the last years due to their potential clinical application: the accessibility of the retina for miRNA-based therapeutic delivery has great potential for the prevention and treatment of retinal pathologies [152]. Up to now, there have only been a handful of studies on miRNA and its role in myopiagenesis in humans, these are summarized in Table 5.6.

\subsection{Implications for Clinical Management}

Due to the high polygenicity of myopia and low explained phenotypic variance by genetic factors $(7.8 \%)$, clinical applications derived from genetic analyses of myopia are currently limited. Risk predictions for myopia in children are based on 
family history, education level of the parents, the amount of outdoor exposure and the easily measurable refractive error and axial length.

Currently, we are able to make a distinction between high myopes and high hyperopes based on the polygenic risk scores derived from CREAM studies: persons in the highest decile for the polygenic risk score had a 40-fold-greater risk of myopia relative to those in the lowest decile.

A prediction model including age, sex and polygenic risk score achieved an AUC of $0.77(95 \% \mathrm{CI}=0.75-0.79)$ for myopia versus hyperopia in adults (Rotterdam Study I-III) [18]. This AUC is similar to that achieved by modelling environmental factors only; the AUC for myopia incidence in a European child cohort was 0.78 considering parental myopia, 1 or more books read per week, time spent reading, no participation in sports, non-European ethnicity, less time spent outdoors and baseline AL-to-CR ratio [156]. To date, one study has assessed both environmental and

Table 5.6 Overview of microRNAs associated with myopia

\begin{tabular}{|c|c|c|c|c|}
\hline MiRNA & SNP & Study design & Outcome & Author \\
\hline $\begin{array}{l}\text { MiR-328 } \\
\text { binding } \\
\text { site in } \\
3^{\prime} \text { UTR } \\
\text { of PAX6 } \\
\text { gene }\end{array}$ & rs662702 & $\begin{array}{l}\text { High myopia case-control } \\
\text { study (Ncase = 1083, } \\
\leq-6 \text { D; Ncontrol } 1096 \\
\geq-1.5 \text { D) }\end{array}$ & $\begin{array}{l}\text { Down regulation effect on } \\
\text { PAX6 expression with } \mathrm{C} \\
\text { allele, relative to T allele. } \\
\text { OR for CC genotype } 2.1 \\
(P=0.007) \text {. This effect } \\
\text { was significant for } \\
\text { extreme myopia } \\
(<-10 \mathrm{D}) \text { and not for high } \\
\text { myopia }\end{array}$ & $\begin{array}{l}\text { Liang } \\
\text { et al. } \\
(2011) \\
{[153]}\end{array}$ \\
\hline MiR-184 & n.a. & $\begin{array}{l}\text { MiR-184 region sequenced } \\
\text { in } 780 \text { unrelated keratoconus } \\
\text { patients and } 96 \text { unrelated } \\
\text { Han southern Chinese } \\
\text { patients with axial myopia } \\
\text { under the hypothesis that } \\
\text { axial myopia is associated } \\
\text { with keratoconus, possibly } \\
\text { under regulation of } \\
\text { MiR-184 }\end{array}$ & $\begin{array}{l}\text { No miR-184 mutations } \\
\text { were detected in the axial } \\
\text { myopia cohort }\end{array}$ & $\begin{array}{l}\text { Lechner } \\
\text { et al. } \\
(2013) \\
{[154]}\end{array}$ \\
\hline MiR-29a & rs 157907 & $\begin{array}{l}\text { High myopia case-control } \\
\text { study (Ncases }=254, \\
\leq-6 \mathrm{D} ; \text { Ncontrols }=300, \\
-0.5 \text { to } 0.5 \mathrm{D}) . \text { COL } 1 \mathrm{~A} 1 \text { is } \\
\text { possibly targeted by } \\
\text { MiR-29a }\end{array}$ & $\begin{array}{l}\text { The G allele of the } \\
\text { rs } 157907 \text { locus was } \\
\text { significantly associated } \\
\text { with decreased risk of } \\
\text { severe myopia (<10 D; } \\
P=0.04 \text { ), compared to } \\
\text { the A allele. rs } 157907 \\
\text { A/G might regulate } \\
\text { miR-29a expression } \\
\text { levels, but no functional } \\
\text { studies have been } \\
\text { conducted to confirm this } \\
\text { hypothesis }\end{array}$ & $\begin{array}{l}\text { Xie } \\
\text { et al. } \\
(2016) \\
{[155]}\end{array}$ \\
\hline Let-7i & rs10877885 & $\begin{array}{l}\text { High myopia case-control } \\
\text { study (Ncases }=254, \\
\leq-6 \mathrm{D} ; \text { Ncontrols }=300 \text {, } \\
-0.5 \text { to } 0.5 \mathrm{D}) . \text { COL1A } 1 \text { is } \\
\text { possibly targeted by Let- } 7 \mathrm{i}\end{array}$ & $\begin{array}{l}\text { No significant association } \\
\text { with rs } 10877885(\mathrm{C} / \mathrm{T}) \\
\text { was found with myopia } \\
\text { risk }\end{array}$ & $\begin{array}{l}\text { Xie } \\
\text { et al. } \\
(2016) \\
{[155]}\end{array}$ \\
\hline
\end{tabular}


genetic factors together and showed that modelling both genes and environment improved prediction accuracy [157]. Although these efforts to improve prediction are promising, a prediction-based approach will only be beneficial if randomized controlled trials of atropine therapy show that children with persistent myopic progression benefit from an earlier and higher dose of atropine administration. The additional costs of genetic testing and potentially invasive regime (collecting blood from children) also need to be taken into account.

\subsection{Concluding Remarks}

The scientific community has discovered more than 200 loci associated with myopia and its endophenotypes with a variety of approaches (linkage, candidate gene, GWAS, post-GWAS gene-based associations, next generation sequencing approaches, gene environment interactions and epigenetic approaches). With the rise of large biobanks, such as the UK Biobank [158], further GWAS meta-analyses between large consortia and companies will enable identification of many more genes. This will allow full elucidation of the molecular mechanism of myopiagenesis. Whole genome sequencing approaches will replace both GWAS and WES, and will elucidate the genetic structure which regulates the function of the myopia risk variants.

To fully understand the underlying mechanisms, the focus should lie on unraveling the genetic and epigenetic architecture of myopia by exploring interactions and effects of other "omics" in relevant tissue, i.e. multi-omics. This concept includes incorporation of methylomics, transcriptomics, proteomics and metabolomics. Future projects should focus on gathering more omics data on eye tissue. Next to the multi-omics approach, modelling gene-environment effects will tell us more about the genetic key players which are also susceptible to the environment. Furthermore, future functional studies interrogating the candidate genes and loci will point us to therapeutic solutions for myopia management.

\section{References}

1. Tedja MS, et al. International Myopia Institute (IMI) - myopia genetics report. Investig Ophthalmol Vis Sci. 2019;60:M31-88.

2. Duke-Elder SS. The practice of refraction. Philadelphia: Blakiston; 1943.

3. Guggenheim JA. The heritability of high myopia: a reanalysis of Goldschmidt's data. J Med Genet. 2000;37:227-31.

4. Sorsby A, Sheridan M, Leary GA. Refraction and its components in twins. Medical Research Council, Special Report Series No. 303; 1962.

5. Lin LL, Chen CJ. A twin study on myopia in Chinese school children. Acta Ophthalmol Suppl. 1988;185:51-3.

6. Wojciechowski R, et al. Heritability of refractive error and familial aggregation of myopia in an elderly American population. Invest Ophthalmol Vis Sci. 2005;46:1588-92.

7. Young FA, et al. The transmission of refractive errors within Eskimo families. Am J Optom Arch Am Acad Optom. 1969;46:676-85.

8. Angi MR, Clementi M, Sardei C, Piattelli E, Bisantis C. Heritability of myopic refractive errors in identical and fraternal twins. Graefes Arch Clin Exp Ophthalmol. 1993;231:580-5.

9. Teikari JM, Kaprio J, Koskenvuo MK, Vannas A. Heritability estimate for refractive errorsDOUBLEHYPHENa population-based sample of adult twins. Genet Epidemiol. 1988;5:171-81. 
10. Lyhne N, Sjølie AK, Kyvik KO, Green A. The importance of genes and environment for ocular refraction and its determiners: a population based study among 20-45 year old twins. Br J Ophthalmol. 2001;85:1470-6.

11. Sanfilippo PG, Hewitt AW, Hammond CJ, Mackey DA. The heritability of ocular traits. Surv Ophthalmol. 2010;55:561-83.

12. OMIM - Online Mendelian Inheritance in Man. Available at https://www.omim.org/. Accessed 27 June 2018.

13. Hendriks M, et al. Development of refractive errors-what can we learn from inherited retinal dystrophies? Am J Ophthalmol. 2017;182:81-9.

14. Iglesias AI, et al. Cross-ancestry genome-wide association analysis of corneal thickness strengthens link between complex and Mendelian eye diseases. Nat Commun. 2018;9:1864.

15. Baratz KH, et al. E2-2 protein and Fuchs's corneal dystrophy. N Engl J Med. 2010;363:1016-24.

16. Mutti DO, et al. Candidate gene and locus analysis of myopia. Mol Vis. 2007;13:1012-9.

17. Metlapally R, et al. COL1A1 and COL2A1 genes and myopia susceptibility: evidence of association and suggestive linkage to the COL2A1 locus. Invest Ophthalmol Vis Sci. 2009;50:4080-6.

18. Tedja MS, et al. Genome-wide association meta-analysis highlights light-induced signaling as a driver for refractive error. Nat Genet. 2018;50:834-48.

19. Fan Q, et al. Meta-analysis of gene-environment-wide association scans accounting for education level identifies additional loci for refractive error. Nat Commun. 2016;7:11008.

20. Flitcroft DI, et al. Novel myopia genes and pathways identified from syndromic forms of myopia. Invest Ophthalmol Vis Sci. 2018;59:338-48.

21. Dawn Teare M, Barrett JH. Genetic linkage studies. Lancet. 2005;366:1036-44.

22. Wojciechowski R. Nature and nurture: the complex genetics of myopia and refractive error. Clin Genet. 2011;79:301-20.

23. Baird PN, Schäche M, Dirani M. The GEnes in Myopia (GEM) study in understanding the aetiology of refractive errors. Prog Retin Eye Res. 2010;29:520-42.

24. Hornbeak DM, Young TL. Myopia genetics: a review of current research and emerging trends. Curr Opin Ophthalmol. 2009;20:356-62.

25. Jacobi FK, Pusch CM. A decade in search of myopia genes. Front Biosci. 2010;15:359-72.

26. Zhang Q, et al. A new locus for autosomal dominant high myopia maps to 4q22-q27 between D4S1578 and D4S1612. Mol Vis. 2005;11:554-60.

27. Young TL, et al. A second locus for familial high myopia maps to chromosome 12q. Am J Hum Genet. 1998;63:1419-24.

28. Naiglin L, et al. A genome wide scan for familial high myopia suggests a novel locus on chromosome 7q36. J Med Genet. 2002;39:118-24.

29. Paluru P, et al. New locus for autosomal dominant high myopia maps to the long arm of chromosome 17. Invest Ophthalmol Vis Sci. 2003;44:1830-6.

30. Nallasamy S, et al. Genetic linkage study of high-grade myopia in a Hutterite population from South Dakota. Mol Vis. 2007;13:229-36.

31. Lam CY, et al. A genome-wide scan maps a novel high myopia locus to $5 \mathrm{p} 15$. Invest Ophthalmol Vis Sci. 2008;49:3768-78.

32. Stambolian D. Genetic susceptibility and mechanisms for refractive error. Clin Genet. 2013;84:102-8.

33. Stambolian D, et al. Genomewide linkage scan for myopia susceptibility loci among Ashkenazi Jewish families shows evidence of linkage on chromosome 22q12. Am J Hum Genet. 2004;75:448-59.

34. Wojciechowski R, et al. Genomewide scan in Ashkenazi Jewish families demonstrates evidence of linkage of ocular refraction to a QTL on chromosome 1p36. Hum Genet. 2006;119:389-99.

35. Wojciechowski R, et al. Genomewide linkage scans for ocular refraction and meta-analysis of four populations in the Myopia Family Study. Invest Ophthalmol Vis Sci. 2009;50:2024-32.

36. Hammond CJ, Andrew T, Mak YT, Spector TD. A susceptibility locus for myopia in the normal population is linked to the PAX6 gene region on chromosome 11: a genomewide scan of dizygotic twins. Am J Hum Genet. 2004;75:294-304.

37. Ciner E, et al. Genome-wide scan of African-American and white families for linkage to myopia. Am J Ophthalmol. 2009;147:512-517.e2. 
38. Hawthorne FA, Young TL. Genetic contributions to myopic refractive error: insights from human studies and supporting evidence from animal models. Exp Eye Res. 2013;114:141-9.

39. Tkatchenko AV, Tkatchenko TV, Guggenheim JA, Verhoeven VJM, Hysi PG, Wojciechowski $\mathrm{R}$, et al. APLP2 regulates refractive error and myopia development in mice and humans. PLoS Genet. 2015;11:e1005432.

40. Liu H-P, Lin Y-J, Lin W-Y, Wan L, Sheu JJ-C, Lin H-J, et al. A novel genetic variant of BMP2K contributes to high myopia. J Clin Lab Anal. 2009;23:362-7.

41. Lin H-J, Kung Y-J, Lin Y-J, Sheu JJC, Chen B-H, Lan Y-C, et al. Association of the lumican gene functional 3'-UTR polymorphism with high myopia. Invest Ophthalmol Vis Sci. 2010;51:96-102.

42. Lin H-J, Wan L, Tsai Y, Chen W-C, Tsai S-W, Tsai F-J. The association between lumican gene polymorphisms and high myopia. Eye. 2010;24:1093-101.

43. Lin H-J, Wan L, Tsai Y, Chen W-C, Tsai S-W, Tsai F-J. Muscarinic acetylcholine receptor 1 gene polymorphisms associated with high myopia. Mol Vis. 2009;15:1774-80.

44. Lin H-J, Wan L, Tsai Y, Liu S-C, Chen W-C, Tsai S-W, et al. Sclera-related gene polymorphisms in high myopia. Mol Vis. 2009;15:1655-63.

45. Khor CC, Grignani R, Ng DPK, Toh KY, Chia K-S, Tan D, et al. cMET and refractive error progression in children. Ophthalmology. 2009;116:1469-74. 1474.e1

46. Inamori Y, Ota M, Inoko H, Okada E, Nishizaki R, Shiota T, et al. The COL1A1 gene and high myopia susceptibility in Japanese. Hum Genet. 2007;122:151-7.

47. Metlapally R, Li Y-J, Tran-Viet K-N, Abbott D, Czaja GR, Malecaze F, et al. COL1A1 and COL2A1 genes and myopia susceptibility: evidence of association and suggestive linkage to the COL2A1 locus. Invest Ophthalmol Vis Sci. 2009;50:4080-6.

48. Mutti DO, Cooper ME, O’Brien S, Jones LA, Marazita ML, Murray JC, et al. Candidate gene and locus analysis of myopia. Mol Vis. 2007;13:1012-9.

49. Ho DWH, Yap MKH, Ng PW, Fung WY, Yip SP. Association of high myopia with crystallin beta A4 (CRYBA4) gene polymorphisms in the linkage-identified MYP6 locus. PLoS One. 2012;7:e40238.

50. Han W, Yap MKH, Wang J, Yip SP. Family-based association analysis of hepatocyte growth factor (HGF) gene polymorphisms in high myopia. Invest Ophthalmol Vis Sci. 2006;47:2291-9.

51. Veerappan S, Pertile KK, Islam AFM, Schäche M, Chen CY, Mitchell P, et al. Role of the hepatocyte growth factor gene in refractive error. Ophthalmology. 2010;117:239-45.e1-2.

52. Yanovitch T, Li Y-J, Metlapally R, Abbott D, Viet K-NT, Young TL. Hepatocyte growth factor and myopia: genetic association analyses in a Caucasian population. Mol Vis. 2009;15:1028-35.

53. Metlapally R, Ki C-S, Li Y-J, Tran-Viet K-N, Abbott D, Malecaze F, et al. Genetic association of insulin-like growth factor-1 polymorphisms with high-grade myopia in an international family cohort. Invest Ophthalmol Vis Sci. 2010;51:4476-9.

54. Zhao YY, Zhang FJ, Zhu SQ, Duan H, Li Y, Zhou ZJ, et al. The association of a single nucleotide polymorphism in the promoter region of the LAMA1 gene with susceptibility to Chinese high myopia. Mol Vis. 2011;17:1003-10.

55. Chen ZT-Y, Wang I-J, Shih Y-F, Lin LL-K. The association of haplotype at the lumican gene with high myopia susceptibility in Taiwanese patients. Ophthalmology. 2009;116:1920-7.

56. Wang I-J, Chiang T-H, Shih Y-F, Hsiao CK, Lu S-C, Hou Y-C, et al. The association of single nucleotide polymorphisms in the 5'-regulatory region of the lumican gene with susceptibility to high myopia in Taiwan. Mol Vis. 2006;12:852-7.

57. Andrew T, Maniatis N, Carbonaro F, Liew SHM, Lau W, Spector TD, et al. Identification and replication of three novel myopia common susceptibility gene loci on chromosome 3q26 using linkage and linkage disequilibrium mapping. PLoS Genet. 2008;4:e1000220.

58. Wojciechowski R, Bailey-Wilson JE, Stambolian D. Association of matrix metalloproteinase gene polymorphisms with refractive error in Amish and Ashkenazi families. Invest Ophthalmol Vis Sci. 2010;51:4989-95.

59. Wojciechowski R, Yee SS, Simpson CL, Bailey-Wilson JE, Stambolian D. Matrix metalloproteinases and educational attainment in refractive error: evidence of gene-environment interactions in the Age-Related Eye Disease Study. Ophthalmology. 2013;120:298-305.

60. Hall NF, Gale CR, Ye S, Martyn CN. Myopia and polymorphisms in genes for matrix metalloproteinases. Invest Ophthalmol Vis Sci. 2009;50:2632-6. 
61. Tang WC, Yip SP, Lo KK, Ng PW, Choi PS, Lee SY, et al. Linkage and association of myocilin (MYOC) polymorphisms with high myopia in a Chinese population. Mol Vis. 2007;13:534-44.

62. Vatavuk Z, Skunca Herman J, Bencić G, Andrijević Derk B, Lacmanović Loncar V, Petric Vicković I, et al. Common variant in myocilin gene is associated with high myopia in isolated population of Korcula Island, Croatia. Croat Med J. 2009;50:17-22.

63. Zayats T, Yanovitch T, Creer RC, McMahon G, Li Y-J, Young TL, et al. Myocilin polymorphisms and high myopia in subjects of European origin. Mol Vis. 2009;15:213-22.

64. Han W, Leung KH, Fung WY, Mak JYY, Li YM, Yap MKH, et al. Association of PAX6 polymorphisms with high myopia in Han Chinese nuclear families. Invest Ophthalmol Vis Sci. 2009;50:47-56.

65. Kanemaki N, Meguro A, Yamane T, Takeuchi M, Okada E, Iijima Y, et al. Study of association of PAX6 polymorphisms with susceptibility to high myopia in a Japanese population. Clin Ophthalmol. 2015;9:2005-11.

66. Miyake M, Yamashiro K, Nakanishi H, Nakata I, Akagi-Kurashige Y, Tsujikawa A, et al. Association of paired box 6 with high myopia in Japanese. Mol Vis. 2012;18:2726-35.

67. Ng TK, Lam CY, Lam DSC, Chiang SWY, Tam POS, Wang DY, et al. AC and AG dinucleotide repeats in the PAX6 P1 promoter are associated with high myopia. Mol Vis. 2009;15:2239-48.

68. Tsai Y-Y, Chiang C-C, Lin H-J, Lin J-M, Wan L, Tsai F-J. A PAX6 gene polymorphism is associated with genetic predisposition to extreme myopia. Eye. 2008;22:576-81.

69. Khor CC, Fan Q, Goh L, Tan D, Young TL, Li Y-J, et al. Support for TGFB1 as a susceptibility gene for high myopia in individuals of Chinese descent. Arch Ophthalmol. 2010;128:1081-4.

70. Lin H-J, Wan L, Tsai Y, Tsai Y-Y, Fan S-S, Tsai C-H, et al. The TGFbetal gene codon 10 polymorphism contributes to the genetic predisposition to high myopia. Mol Vis. 2006;12:698-703.

71. Rasool S, Ahmed I, Dar R, Ayub SG, Rashid S, Jan T, et al. Contribution of TGF $\beta 1$ codon 10 polymorphism to high myopia in an ethnic Kashmiri population from India. Biochem Genet. 2013;51:323-33.

72. Zha Y, Leung KH, Lo KK, Fung WY, Ng PW, Shi M-G, et al. TGFB1 as a susceptibility gene for high myopia: a replication study with new findings. Arch Ophthalmol. 2009;127:541-8.

73. Lam DSC, Lee WS, Leung YF, Tam POS, Fan DSP, Fan BJ, et al. TGFbeta-induced factor: a candidate gene for high myopia. Invest Ophthalmol Vis Sci. 2003;44:1012-5.

74. Ahmed I, Rasool S, Jan T, Qureshi T, Naykoo NA, Andrabi KI. TGIF1 is a potential candidate gene for high myopia in ethnic Kashmiri population. Curr Eye Res. 2014;39:282-90.

75. Nishizaki R, Ota M, Inoko H, Meguro A, Shiota T, Okada E, et al. New susceptibility locus for high myopia is linked to the uromodulin-like 1 (UMODL1) gene region on chromosome 21q22.3. Eye. 2009;23:222-9.

76. Tang SM, et al. PAX6 gene associated with high myopia: a meta-analysis. Optom Vis Sci. 2014;91:419-29.

77. Li M, et al. Lack of association between LUM rs3759223 polymorphism and high myopia. Optom Vis Sci. 2014;91:707-12.

78. Zhang D, et al. Association of IGF1 polymorphism rs6214 with high myopia: a systematic review and meta-analysis. Ophthalmic Genet. 2017;38:434-9.

79. Springelkamp H, et al. New insights into the genetics of primary open-angle glaucoma based on meta-analyses of intraocular pressure and optic disc characteristics. Hum Mol Genet. 2017;26:438-53.

80. Burdon KP, et al. Association of polymorphisms in the hepatocyte growth factor gene promoter with keratoconus. Invest Ophthalmol Vis Sci. 2011;52:8514-9.

81. Risch N, Merikangas K. The future of genetic studies of complex human diseases. Science. 1996;273:1516-7.

82. Ku CS, Loy EY, Pawitan Y, Chia KS. The pursuit of genome-wide association studies: where are we now? J Hum Genet. 2010;55:195-206.

83. Cordell HJ, Clayton DG. Genetic association studies. Lancet. 2005;366:1121-31.

84. Nakanishi $\mathrm{H}$, et al. A genome-wide association analysis identified a novel susceptible locus for pathological myopia at 11q24.1. PLoS Genet. 2009;5:e1000660.

85. Li Y-J, et al. Genome-wide association studies reveal genetic variants in CTNND2 for high myopia in Singapore Chinese. Ophthalmology. 2011;118:368-75. 
86. Lu B, et al. Replication study supports CTNND2 as a susceptibility gene for high myopia. Invest Ophthalmol Vis Sci. 2011;52:8258-61.

87. Wang Q, et al. Replication study of significant single nucleotide polymorphisms associated with myopia from two genome-wide association studies. Mol Vis. 2011;17:3290-9.

88. Yu Z, et al. Polymorphisms in theCTNND2Gene and 11q24.1 genomic region are associated with pathological myopia in a Chinese population. Ophthalmologica. 2012;228:123-9.

89. Liu J, Zhang H-X. Polymorphism in the 11q24.1 genomic region is associated with myopia: a comprehensive genetic study in Chinese and Japanese populations. Mol Vis. 2014;20:352-8.

90. Shi Y, et al. Genetic variants at 13q12.12 are associated with high myopia in the Han Chinese population. Am J Hum Genet. 2011;88:805-13.

91. Shi Y, et al. A genome-wide meta-analysis identifies two novel loci associated with high myopia in the Han Chinese population. Hum Mol Genet. 2013;22:2325-33.

92. Khor CC, et al. Genome-wide association study identifies ZFHX1B as a susceptibility locus for severe myopia. Hum Mol Genet. 2013;22:5288-94.

93. Hosoda Y, et al. CCDC102B confers risk of low vision and blindness in high myopia. Nat Commun. 2018;9:1782.

94. Meng W, et al. A genome-wide association study provides evidence for association of chromosome 8p23 (MYP10) and 10q21.1 (MYP15) with high myopia in the French population. Invest Ophthalmol Vis Sci. 2012;53:7983-8.

95. Pickrell JK, et al. Detection and interpretation of shared genetic influences on 42 human traits. Nat Genet. 2016;48:709-17.

96. Solouki AM, et al. A genome-wide association study identifies a susceptibility locus for refractive errors and myopia at 15q14. Nat Genet. 2010;42:897-901.

97. Hysi PG, et al. A genome-wide association study for myopia and refractive error identifies a susceptibility locus at $15 q 25$. Nat Genet. 2010;42:902-5.

98. Stambolian D, et al. Meta-analysis of genome-wide association studies in five cohorts reveals common variants in RBFOX1, a regulator of tissue-specific splicing, associated with refractive error. Hum Mol Genet. 2013;22:2754-64.

99. Verhoeven VJM, et al. Large scale international replication and meta-analysis study confirms association of the $15 \mathrm{q} 14$ locus with myopia. The CREAM consortium. Hum Genet. 2012;131:1467-80.

100. Schache M, et al. Genetic association of refractive error and axial length with $15 \mathrm{q} 14$ but Not 15q25 in the Blue Mountains Eye Study cohort. Ophthalmology. 2013;120:292-7.

101. Simpson CL, et al. Regional replication of association with refractive error on $15 \mathrm{q} 14$ and 15q25 in the Age-Related Eye Disease Study cohort. Mol Vis. 2013;19:2173-86.

102. Simpson CL, Wojciechowski R, Oexle K, Murgia F, Portas L, Li X, et al. Genome-wide metaanalysis of myopia and hyperopia provides evidence for replication of 11 loci. PLoS One. 2014;9:e107110.

103. Yoshikawa M, Yamashiro K, Miyake M, Oishi M, Akagi-Kurashige Y, Kumagai K, et al. Comprehensive replication of the relationship between myopia-related genes and refractive errors in a large Japanese cohort. Invest Ophthalmol Vis Sci. 2014;55:7343-54.

104. Cheng C-Y, Schache M, Ikram MK, Young TL, Guggenheim JA, Vitart V, et al. Nine loci for ocular axial length identified through genome-wide association studies, including shared loci with refractive error. Am J Hum Genet. 2013;93:264-77.

105. Oishi M, Yamashiro K, Miyake M, Akagi-Kurashige Y, Kumagai K, Nakata I, et al. Association between ZIC2, RASGRF1, and SHISA6 genes and high myopia in Japanese subjects. Invest Ophthalmol Vis Sci. 2013;54:7492-7.

106. Tideman JWL, Fan Q, Polling JR, Guo X, Yazar S, Khawaja A, et al. When do myopia genes have their effect? Comparison of genetic risks between children and adults. Genet Epidemiol. 2016;40:756-66.

107. Liao X, Yap MKH, Leung KH, Kao PYP, Liu LQ, Yip SP. Genetic association study of KCNQ5 polymorphisms with high myopia. Biomed Res Int. 2017;2017:3024156.

108. Verhoeven VJM, et al. Genome-wide meta-analyses of multiancestry cohorts identify multiple new susceptibility loci for refractive error and myopia. Nat Genet. 2013;45:314-8.

109. Wojciechowski R, Hysi PG. Focusing in on the complex genetics of myopia. PLoS Genet. 2013;9:e1003442.

110. Kiefer AK, et al. Genome-wide analysis points to roles for extracellular matrix remodeling, the visual cycle, and neuronal development in myopia. PLoS Genet. 2013;9:e1003299. 
111. Mishra A, et al. Genetic variants near PDGFRA are associated with corneal curvature in Australians. Invest Ophthalmol Vis Sci. 2012;53:7131-6.

112. Guggenheim JA, et al. A genome-wide association study for corneal curvature identifies the platelet-derived growth factor receptor $\alpha$ gene as a quantitative trait locus for eye size in white Europeans. Mol Vis. 2013;19:243-53.

113. Han S, et al. Association of variants in FRAP1 and PDGFRA with corneal curvature in Asian populations from Singapore. Hum Mol Genet. 2011;20:3693-8.

114. Chen P, et al. CMPK1 and RBP3 are associated with corneal curvature in Asian populations. Hum Mol Genet. 2014;23:6129-36.

115. Miyake $M$, et al. Identification of myopia-associated WNT7B polymorphisms provides insights into the mechanism underlying the development of myopia. Nat Commun. 2015;6:6689.

116. Hysi PG, et al. Common mechanisms underlying refractive error identified in functional analysis of gene lists from genome-wide association study results in 2 European British cohorts. JAMA Ophthalmol. 2014;132:50-6.

117. Chandra A, Mitry D, Wright A, Campbell H, Charteris DG. Genome-wide association studies: applications and insights gained in ophthalmology. Eye. 2014;28:1066-79.

118. Hysi PG, Wojciechowski R, Rahi JS, Hammond CJ. Genome-wide association studies of refractive error and myopia, lessons learned, and implications for the future. Invest Ophthalmol Vis Sci. 2014;55:3344-51.

119. Napolitano F, et al. Autosomal-dominant myopia associated to a novel P4HA2 missense variant and defective collagen hydroxylation. Clin Genet. 2018;93:982-91.

120. Zhao F, et al. Exome sequencing reveals CCDC111 mutation associated with high myopia. Hum Genet. 2013;132:913-21.

121. Wang B, et al. A novel potentially causative variant of NDUFAF7 revealed by mutation screening in a Chinese family with pathologic myopia. Invest Ophthalmol Vis Sci. 2017;58:4182-92.

122. Feng L, et al. Exome sequencing identifies a novel UNC5D mutation in a severe myopic anisometropia family: a case report. Medicine. 2017;96:e7138.

123. Jiang D, et al. Detection of mutations in LRPAP1, CTSH, LEPREL1, ZNF644, SLC39A5, and SCO2 in 298 families with early-onset high myopia by exome sequencing. Invest Ophthalmol Vis Sci. 2014;56:339-45.

124. Tran-Viet K-N, et al. Mutations in SCO2 are associated with autosomal-dominant high-grade myopia. Am J Hum Genet. 2013;92:820-6.

125. Jin $\mathrm{ZB}$, et al. Trio-based exome sequencing arrests de novo mutations in early-onset high myopia. Proc Natl Acad Sci U S A. 2017;114:4219-24.

126. Xiao X, Li S, Jia X, Guo X, Zhang Q. X-linked heterozygous mutations in cause femalelimited early onset high myopia. Mol Vis. 2016;22:1257-66.

127. Li J, et al. Exome sequencing identified null mutations in LOXL3 associated with early-onset high myopia. Mol Vis. 2016;22:161-7.

128. Guo H, et al. SLC39A5 mutations interfering with the BMP/TGF- $\beta 1$ pathway in non-syndromic high myopia. J Med Genet. 2014;51:518-25.

129. Aldahmesh MA, et al. Mutations in LRPAP1 are associated with severe myopia in humans. Am J Hum Genet. 2013;93:313-20.

130. Shi Y, et al. Exome sequencing identifies ZNF644 mutations in high myopia. PLoS Genet. 2011;7:e1002084.

131. Kloss BA, et al. Exome sequence analysis of 14 families with high myopia. Invest Ophthalmol Vis Sci. 2017;58:1982-90.

132. Sun W, et al. Exome sequencing on 298 probands with early-onset high myopia: approximately one-fourth show potential pathogenic mutations in RetNet genes. Invest Ophthalmol Vis Sci. 2015;56:8365-72.

133. Morgan I, Rose K. How genetic is school myopia? Prog Retin Eye Res. 2005;24:1-38.

134. Morgan IG, Ohno-Matsui K, Saw S-M. Myopia. Lancet. 2012;379:1739-48.

135. Dolgin E. The myopia boom. Nature. 2015;519:276-8.

136. Williams KM, et al. Increasing prevalence of myopia in europe and the impact of education. Ophthalmology. 2015;122:1489-97.

137. Dirani M, Shekar SN, Baird PN. The role of educational attainment in refraction: the Genes in Myopia (GEM) twin study. Invest Ophthalmol Vis Sci. 2008;49:534-8.

138. Foster PJ, Jiang Y. Epidemiology of myopia. Eye. 2014;28:202-8. 
139. Pan C-W, Ramamurthy D, Saw S-M. Worldwide prevalence and risk factors for myopia. Ophthalmic Physiol Opt. 2012;32:3-16.

140. Mirshahi A, et al. Myopia and level of education: results from the Gutenberg Health Study. Ophthalmology. 2014;121:2047-52.

141. Morgan IG, Rose KA. Myopia and international educational performance. Ophthalmic Physiol Opt. 2013;33:329-38.

142. Ramessur R, Williams KM, Hammond CJ. Risk factors for myopia in a discordant monozygotic twin study. Ophthalmic Physiol Opt. 2015;35:643-51.

143. Fan Q, et al. Education influences the association between genetic variants and refractive error: a meta-analysis of five Singapore studies. Hum Mol Genet. 2014;23:546-54.

144. Verhoeven VJM, et al. Education influences the role of genetics in myopia. Eur J Epidemiol. 2013;28:973-80.

145. Fan Q, et al. Childhood gene-environment interactions and age-dependent effects of genetic variants associated with refractive error and myopia: The CREAM Consortium. Sci Rep. 2016;6:25853.

146. Ebrahim S, Davey Smith G. Mendelian randomization: can genetic epidemiology help redress the failures of observational epidemiology? Hum Genet. 2008;123:15-33.

147. Smith GD, Ebrahim S. Mendelian randomization: prospects, potentials, and limitations. Int J Epidemiol. 2004;33:30-42.

148. Mountjoy E, et al. Education and myopia: assessing the direction of causality by mendelian randomisation. BMJ. 2018;362:k2932.

149. Cuellar-Partida G, et al. Assessing the genetic predisposition of education on myopia: A Mendelian Randomization Study. Genet Epidemiol. 2016;40:66-72.

150. Cuellar-Partida G, et al. Genetically low vitamin D concentrations and myopic refractive error: a Mendelian randomization study. Int J Epidemiol. 2017;46:1882-90.

151. Teperino R, Lempradl A, Pospisilik JA. Bridging epigenomics and complex disease: the basics. Cell Mol Life Sci. 2013;70:1609-21.

152. Jiang B, Huo Y, Gu Y, Wang J. The role of microRNAs in myopia. Graefes Arch Clin Exp Ophthalmol. 2017;255:7-13.

153. Liang CL, et al. A functional polymorphism at 3'UTR of the PAX6 gene may confer risk for extreme myopia in the Chinese. Invest Ophthalmol Vis Sci. 2011;52:3500-5.

154. Lechner J, et al. Mutational analysis of MIR184 in sporadic keratoconus and myopia. Invest Ophthalmol Vis Sci. 2013;54:5266-72.

155. Xie M, et al. Genetic variants in MiR-29a associated with high myopia. Ophthalmic Genet. 2016;37:456-8.

156. Tideman JWL, et al. Environmental factors explain socioeconomic prevalence differences in myopia in 6-year-old children. Br J Ophthalmol. 2018;102:243-7.

157. Ghorbani Mojarrad N, Williams C, Guggenheim JA. A genetic risk score and number of myopic parents independently predict myopia. Ophthalmic Physiol Opt. 2018;38(5):492_ 502. https://doi.org/10.1111/opo.12579.

158. Sudlow C, et al. UK biobank: an open access resource for identifying the causes of a wide range of complex diseases of middle and old age. PLoS Med. 2015;12:e1001779.

Open Access This chapter is licensed under the terms of the Creative Commons Attribution 4.0 International License (http://creativecommons.org/licenses/by/4.0/), which permits use, sharing, adaptation, distribution and reproduction in any medium or format, as long as you give appropriate credit to the original author(s) and the source, provide a link to the Creative Commons license and indicate if changes were made.

The images or other third party material in this chapter are included in the chapter's Creative Commons license, unless indicated otherwise in a credit line to the material. If material is not included in the chapter's Creative Commons license and your intended use is not permitted by statutory regulation or exceeds the permitted use, you will need to obtain permission directly from the copyright holder. 